\title{
An Adaptive Procedure for the Numerical Parameters of a Particle Simulation
}

\author{
Cyril Galitzine ${ }^{\mathrm{a}, *}$, Iain D. Boyd ${ }^{\mathrm{a}}$ \\ ${ }^{a}$ University of Michigan, Department of Aerospace Engineering, Ann Arbor, MI 48109, United States
}

\begin{abstract}
In this article, a computational procedure that automatically determines the optimum time step, cell weight and species weights for steady-state multi-species DSMC (direct simulation Monte Carlo) simulations is presented. The time step is required to satisfy the basic requirements of the DSMC method while the weight and relative weights fields are chosen so as to obtain a user-specified average number of particles in all cells of the domain. The procedure allows the conduct of efficient DSMC simulations with minimal user input and is integrable into existing DSMC codes. The adaptive method is used to simulate a test case consisting of two counterflowing jets at a Knudsen number of 0.015. Large accuracy gains for sampled number densities and velocities over a standard simulation approach for the same number of particles are observed.
\end{abstract}

Keywords: DSMC, direct simulation Monte Carlo, weight, time step, species weighting

\section{Introduction}

The DSMC (direct simulation Monte Carlo) method is today the most widely used simulation method for high speed rarefied flows [1]. Its accuracy and convergence is mainly determined by the number of particles employed in the simulation, the mesh size and the time step employed. Because of the formulation of DSMC [2], the number of particles in a cell is directly proportional to its number density while the time step of the simulation can be no more than the mean collision time so that the movement and collisions of particles can be decoupled. Similarly, collisions are only performed for particles located in the same cell, so that its characteristic length has to be less than the mean free path for this local assumption to be valid. This in turn signifies that the constraints which the numerical parameters of DSMC have to satisfy are inherently tied to the physics, particularly the number density field, of the test case being simulated. This, however, makes the efficient simulation of flows containing large variations in number density or large disparities between species number densities difficult. Large density variations are commonly encountered in many rarefied flows, particularly jets, while the accurate simulation of trace species, i.e. chemical species with a low concentration relative to others, is important for many applications. This is in particular the case for weakly ionized flows where the electric field is determined by the distribution of charged particles whose concentrations are low compared to neutral particles [3] [4]. Trace species also play an important role in chemically reacting [5] and radiating [6] flows. These drawbacks inherent to the DSMC method have led to a number of modifications to the basic DSMC algorithm that are in ubiquitous use today. The first is the use of spatially varying weights, particularly for axisymmetric simulations, such as in (amongst many others) [7] [8] where the cell volume scales with the distance to the centerline, that allows enough particles to be present close to the centerline while reducing their number further away from it. The second improvement is the use of a spatially varying time step, and an adaptive procedure to calculate the optimum

\footnotetext{
* Corresponding author.

Email address: cyrilg@umich.edu (Cyril Galitzine)
} 
time step field as in, e.g. , [9] [10] [11]. For multi-species flow, the use of relative weights, also called species weights, is widespread, such as in [6] [12] [13]. They allow to increase the number of particles representing species with low number densities relative to others thereby allowing the use of fewer particles overall in the simulation. Spatially varying time steps, cell weights and relative weights are, however, almost never used simultaneously and whereas an adaptive procedure for the time step has been proposed before [9] [10], one for the cell weights and species relative weights has not. Relative weights are furthermore most often assumed to be spatially uniform thereby forfeiting some of the efficiency gains obtainable by having them vary through the computational domain. The aim of this article is to detail a formulation of an allencompassing adaptive procedure for the time step, cell weights and relative weights where all are allowed to vary throughout space. Such a procedure greatly facilitates the conduct of efficient DSMC simulations by lessening the need for human inputs, such as e.g. , running multiple simulations to determine optimum weight fields. The first part of the article describes the additional considerations that must be taken into account when using spatially varying weights or time steps in terms of particle movement and collisions including the potential deleterious effects of particle cloning. The adaptive procedure for the time step, cell weight and species relative weights is then detailed and its integration into existing DSMC codes discussed. A test case consisting of two counter-flowing axisymmetric jets at a Knudsen number of 0.015 is introduced which is used to illustrate the increased accuracy obtainable with the adaptive method compared to when only a spatially varying time step and weight are used.

\section{DSMC Framework}

\subsection{DSMC with spatially varying time step and weights}

\subsubsection{Definitions}

In the canonical version of DSMC, the same cell weight $W_{p, 0}$ and time step value $\Delta t_{0}$ are used for all $N_{c}$ cells of the computational domain while all species are assigned the same relative weight of 1 . In the following, spatially variable cellwise constant time step $\Delta t(\vec{x})$, cell weights $W_{p}(\vec{x})$ and species relative weights $W_{\text {rel, }, j}(\vec{x})$ are considered. A non-dimensional time step $\widetilde{\Delta} t(\vec{x})$ and cell weight $\widetilde{W}_{p}(\vec{x})$ are in turn, respectively, defined as

$$
\Delta t(\vec{x})=\Delta t_{0} \widetilde{\Delta t}(\vec{x})
$$

$$
W_{p}(\vec{x})=W_{p, 0} \widetilde{W}_{p}(\vec{x}) \widetilde{\Delta t}(\vec{x})
$$

The non-dimensionalized weight $\widetilde{W}_{p}(\vec{x})$ is defined as such because it will later be more useful to consider the value of the normalized value of the weight divided by the non-dimensionalized time step, i.e. $\frac{W_{p}(\vec{x})}{W_{p, 0} \widetilde{\Delta t}(\vec{x})}$ instead of $\widehat{W}_{p}(\vec{x}) \triangleq \frac{W_{p}(\vec{x})}{W_{p, 0}}$. Cellwise constant weights and time step are used, while a total of $N_{\text {spec }}$ distinct species are present in the simulation, so that each cell $i\left(1 \leq i \leq N_{c}\right)$ is characterized by $\widetilde{\Delta t}_{i}, \widetilde{W}_{p, i}$ and $\left\{W_{\text {rel }, j, i}\right\}_{j=1}^{N_{\text {spec }}}$. As an example, using the previously defined terminology, the number density $n_{j, i}$ of species $j$ inside cell $i$ of volume $V_{i}$, when it contains $N_{j, i}$ particles of species $j$, is given by:

$$
n_{j, i}=\frac{W_{p, 0} W_{\mathrm{rel}, j, i} \widetilde{W}_{p, i} \widetilde{\Delta t}{ }_{i} N_{j, i}}{V_{i}}
$$

\subsubsection{Particle Movement}

When a computational particle of species $j$ moves from one cell $i$, characterized $\widetilde{W}_{p, i}, W_{\text {rel }, j, i}, \widetilde{\Delta t} i$ to cell $i+1$ with different weights $\widetilde{W}_{p, i+1}, W_{\mathrm{rel}, j, i+1}$ and a different timestep $\widetilde{\Delta t}_{i+1}$, care must be taken to preserve the flux of particles between the two cells. The number density flux (i.e. the number of physical particles per unit area and time) from cell $i$ to cell $i+1$ through their common face of area $S_{i+1 / 2}$ from the standpoint of cell $i$ is denoted by $\Phi_{i \rightarrow i+1 \mid i}^{k}\left(n_{j}\right)$ and given as follows:

$$
\Phi_{i \rightarrow i+1 \mid i}^{k}\left(n_{j}\right)=\frac{W_{\mathrm{rel}, j, i} W_{p, i}}{\Delta t_{i} S_{i+1 / 2}} N_{j, i \rightarrow i+1 \mid i}^{k}=\frac{W_{p, 0}}{\Delta t_{0} S_{i+1 / 2}} W_{\mathrm{rel}, j, i} \widetilde{W}_{p, i} N_{j, i \rightarrow i+1 \mid i}^{k},
$$


where $N_{j, i \rightarrow i+1 \mid i}^{k}$ designates the number of computational particles moving from cell $i$ to cell $i+1$. Similarly, the number density flux of incoming particles from cell $i$ into cell $i+1$ from the standpoint of cell $i+1$ is

$$
\Phi_{i \rightarrow i+1 \mid i+1}\left(n_{j}\right)=\frac{W_{\mathrm{rel}, j, i+1} W_{p, i+1}}{\Delta t_{i+1} S_{i+1 / 2}} N_{j, i \rightarrow i+1 \mid i+1}^{k}=\frac{W_{p, 0}}{\Delta t_{0} S_{i+1 / 2}} W_{\mathrm{rel}, j, i+1} \widetilde{W}_{p, i+1} N_{j, i \rightarrow i+1 \mid i+1}^{k} .
$$

As the number density flux from cell $i$ to cell $i+1$ is a physical quantity, its value cannot be dependent on the value of the numerical parameters used to express it or their spatial distributions, that is

$$
\Phi_{i \rightarrow i+1 \mid i}^{k}\left(n_{j}\right)=\Phi_{i \rightarrow i+1 \mid i+1}^{k}\left(n_{j}\right) \forall\left\{W_{p, i^{\prime}}\right\}_{i^{\prime}},\left\{W_{\mathrm{rel}, j, i^{\prime}}\right\}_{i^{\prime}},\left\{\Delta t_{i^{\prime}}\right\}_{i^{\prime}},
$$

so that:

$$
\frac{W_{p, 0}}{\Delta t_{0} S_{i+1 / 2}} W_{\mathrm{rel}, j, i} \widetilde{W}_{p, i} N_{j, i \rightarrow i+1 \mid i}^{k}=\frac{W_{p, 0}}{\Delta t_{0} S_{i+1 / 2}} W_{\mathrm{rel}, j, i+1} \widetilde{W}_{p, i+1} N_{j, i \rightarrow i+1 \mid i+1}^{k} .
$$

Eq. (7) can be satisfied for all weights and time step distributions if we allow the number of particles moving into cell $i+1$ from the standpoint of cell $i+1$ to depend on them, that is:

$$
N_{j, i \rightarrow i+1 \mid i+1}^{k}=\frac{\widetilde{W}_{p, i} W_{\mathrm{rel}, j, i}}{\widetilde{W}_{p, i+1} W_{\mathrm{rel}, j, i+1}} N_{j, i \rightarrow i+1 \mid i}^{k}
$$

In terms of particles, this in turn signifies, that one particle (corresponding to $N_{j, i \rightarrow i+1 \mid i}^{k}=1$ ) entering cell $i+1$ from cell $i$, needs to give rise to $R_{j, i \rightarrow i+1} \triangleq \frac{\widetilde{W}_{p, i} W_{\mathrm{rel}, j, i}}{\widetilde{W}_{p, i+1} W_{\mathrm{rel}, j, i+1}}$ particles immediately upon entry into cell $i+1$. When $R_{j, i \rightarrow i+1}<1$, the particle is discarded upon reaching the interface between the two cells with a probability of $R_{j, i \rightarrow i+1}$. When $R_{j, i \rightarrow i+1}>1$, the original particle moves to cell $i+1$ and an additional $\left(N_{j, i \rightarrow i+1 \mid i+1}^{k}-1\right)$ particles are generated at the interface following a uniform distribution $\mathcal{U}\left(R_{j, i \rightarrow i+1}-1, R_{j, i \rightarrow i+1}\right)$. The simplest approach and that adopted in this work is to give properties (i.e. position and velocity) that are identical to those of the original particle to all newly generated particles. When that scheme is used, the new particles are then referred to as clones and the particle creation process as cloning. The number of particles that are either generated or destroyed due to the spatial distribution of the weights and the time step is thus controlled by the spatial distribution of $\widetilde{W}_{p, i}$, instead of that of $W_{p, i}$. To be able to more closely control the distribution of $\widetilde{W}_{p, i}$, the adaptive procedure presented in Section 3 is thus formulated to adapt the values of $\widetilde{W}_{p, i}$ (instead of $\widetilde{\Delta} t$ and $\hat{W}_{p, i}$ as might have been expected). An alternate cloning procedure, described in e.g. [2] p. 372 can also be used so as to mitigate the deleterious effect of the standard cloning procedure which is described in the next section. In such, the $\left(N_{j, i \rightarrow i+1}^{k}-1\right)$ cloned particles are introduced in cell $i+1$ over the course of multiple time steps with the aim of promoting collisions between newly created clones and other particles. In view of the additional complexity that such a procedure introduces and given the satisfactory results obtained, it was however not used in the current work.

\subsection{Cloning issues}

The generation of clone particles at the interface between cells where $R_{j, i \rightarrow i+1}>1$ does lead to several issues with consequences that will later become apparent in simulations.

\subsubsection{Accuracy}

It is no better in terms of accuracy for a particular cell to contain two identical particles on average than for it to contain only one. This is because, absent any collisions, cloning does not increase the flow of information traveling from the rest of the computational domain to a particular cell. It only amplifies information by creating more vectors to carry it (i.e. particles). This is the broad reason why increasing the average particle count in a cell through cloning, while striving to maintain the same average number of particles in all cells of the domain, never produces as accurate results as are obtained by increasing the overall total number of particles in the simulation so as to obtain the exact same average particle 
count in that cell. The situation does change when collisions occur as the collision of cloned particles with other particles does generate new information (i.e. new velocities for some of the cloned particles). This is the reason why the present adaptive procedure will tend to produce the greatest relative accuracy gains compared to a simulation employing uniform weights for the same computational cost for flows that are relatively collisional. Such collisions, however, have to occur between non-clone particles, as two identical particles have the same velocity and thus can never collide. In the limiting case of a fully free molecular flow, a simulation with spatially varying weights and time step will produce nearly identical results to those obtained when both are uniform. In extremely collisional flows, the time between the creation of a cloned particle and its first collision is expected to be so short that the deleterious effects of introducing cloned particles should be very small.

\subsubsection{Avalanche phenomenon}

The relative absence of collisions and the use of decreasing weights to counterbalance a decrease in number density along one direction can both combine to create what we refer to as a particle avalanche phenomenon. It occurs when an ever increasing number of cloned particles are created as an increasingly large group, a packet, of identical particles travels along a direction with decreasing weights. A simplified analytical illustration of the phenomenon within the framework of a one-dimensional cold gas is presented in the Appendix at the end of the article. If collisions with other non-identical particles of the same species or with other species are not prevalent enough to break up the packet of particles, the avalanching phenomenon will occur. This undesirable phenomenon will later be seen to limit the useful range of applicability of the adaptive procedure.

\subsection{Average quantities}

The adaptive procedure presented in this article aims to vary the value of the weights so that all cells contain the same approximate number of particles on average as the instantaneous number of particles fluctuates. The average value needs to be reasonably responsive to changes in particle counts that are caused by the application of the adaptive procedure so that an exponential moving average [14] is used for its calculation:

$$
\bar{N}_{j, i}^{k}=R N_{j, i}^{k}+(1-R) \bar{N}_{j, i}^{k-1},
$$

where $N_{j, i}^{k}$ and $\bar{N}_{j, i}^{k}$ are, respectively, the instantaneous number and the average number of particles of species $j$ in cell $i$ at time step $k$. A value of 0.001 for the relaxation factor $R$ is used for this present work. The average number of collision $\bar{N}_{\text {coll }, i}^{k}(a, b)$ between species $a$ and $b$ in cell $i$ at time step $k$ is also similarly calculated as follows:

$$
\bar{N}_{\mathrm{coll}, i}^{k}(a, b)=R N_{\mathrm{coll}, i}^{k}(a, b)+(1-R) \bar{N}_{\mathrm{coll}, i}^{k-1}(a, b),
$$

where $N_{\mathrm{coll}, i}^{k}(a, b)$ is the number of particles in cell $i$ of species $a$ that see their velocity changed as the result of a collision with a particle of species $b$. As will later be discussed in Section 2.5, when species $a$ and $b$ have different relative weights, $N_{\text {coll }, i}^{k}(a, b) \neq N_{\text {coll }, i}^{k}(b, a)$. The average number of collisions is used to calculate the average mean collision time, $\bar{\tau}_{m, i}^{k}(a, b)$, in each cell $i$ following:

$$
\bar{\tau}_{m, i}^{k}(a, b)=\frac{\Delta t \bar{N}_{b, i}^{k}}{\left(1+\delta_{a b}\right) \bar{N}_{\mathrm{coll}, i}^{k}(a, b)},
$$

where $\delta_{a b}$ is the Kronecker delta. $\bar{\tau}_{m, i}^{k}(a, b)$ corresponds to the average time elapsed between two collisions for a particle of species $a$ with one of species $b$ where the velocity of the former is indeed changed. As will later be seen, we are most interested in the minimum value of $\bar{\tau}_{m, i}^{k}(a, b)$ over all species pairs, which is denoted by $\bar{\tau}_{m, i}^{k}$. It corresponds to the mean collision time between the two species that collide with the greatest frequency:

$$
\bar{\tau}_{m, i}^{k}=\min _{a, b} \bar{\tau}_{m, i}^{k}(a, b)
$$

which has to be determined for each individual cell. 


\subsection{Collision rates}

The expression for the number of potential collision pairs to consider between different species with distinct relative weights is given without derivation in [6] (Eq. (5) therein which has a typographic error). Because the derivation is not straightforward, it is repeated here by distinguishing between the cross section of the physical particles and that of the computational particles [15]. This allows a much more rigorous derivation and presentation of all DSMC quantities related to collisions. The DSMC procedure can be viewed and examined from many different viewpoints, e.g. as a stochastic process that mimics the Boltzmann equation [16] or by adopting a more physical viewpoint, as we choose to do here, as a computational gas composed of computational particles. The properties of the computational gas such as its average number density over a specified region of space differ from those of the actual gas. That is, all quantities related to the computational gas have to be suitably rescaled to obtain those of the physical gas that is being modeled. Following the arguments presented in p. 7-8 of [2], the collision frequency of a single particle of the physical gas of species $a$ with particles of gas of species $b$ at a number density of $n_{b}$ is given by:

$$
\nu_{a b}^{(1)}=n_{b} \overline{\sigma_{a b} c_{r}},
$$

where $c_{r}$ is the relative velocity between particles and $\sigma_{a b}$ is the cross section for elastic collisions between species $a$ and $b$. The bar over a quantity designates its average value amongst all particles of the sample. The properties of the DSMC gas differ from those of the physical gas and are denoted with a tilde. In the simulation, species $b$ has a number density of

$$
\tilde{n}_{b}=\frac{n_{b}}{W_{p} W_{\mathrm{rel}, b}}
$$

as each macro or computational particle represents $W_{p} W_{\text {rel }, b}$ actual particles. Similarly, the single particle collision rate of the numerical gas is denoted by $\tilde{\nu}_{a b}^{(1)}$. In the rest of this section, we will drop for clarity the $i$ cell subscript with the understanding that all the expressions in the following are valid for each particular cell of the domain with weight $W_{p}$, relative weights $\left\{W_{\text {rel }, j}\right\}_{j=1}^{N_{\text {spec }}}$, time step $\Delta t$ and a volume of $V$. The particles of the numerical gas have a collisional cross section that is different than those of the actual physical particles. It is denoted by $\tilde{\sigma}_{a b}$ to distinguish it from $\sigma_{a b}$. Equation (13) correspondingly yields the single particle numerical collision rate:

$$
\tilde{\nu}_{a b}^{(1)}=\tilde{n}_{b} \bar{\sigma}_{a b} \tilde{c}_{r} .
$$

The DSMC procedure is based on the fundamental assumption that all particle-carried quantities (e.g. velocity, position, internal energy) are identical between the physical and computational gases. They thus share the same velocity, so that $\tilde{c}_{r}=c_{r}$ as well as the same collision rate which implies that $\nu_{a b}^{(1)}=\tilde{\nu}_{a b}^{(1)}$. Thus, equaling Eqs. (13) and (15) and using Eq. (14):

$$
n_{b} \overline{\sigma_{a b} c_{r}}=\frac{n_{b}}{W_{\mathrm{rel}, b} W_{p}} \overline{\tilde{\sigma}_{a b} c_{r}},
$$

the computational cross section $\tilde{\sigma}$ is thus found to be given by:

$$
\tilde{\sigma}_{a b}=W_{\mathrm{rel}, b} W_{p} \sigma_{a b} .
$$

The probability of a collision, $P_{\text {coll }}\left(a^{\prime}, b^{\prime}\right)$, between a moving particle $a^{\prime}$ and a target particle $b^{\prime}$ belonging, respectively, to species $a$ and $b$, both located inside the same cell of volume $\mathrm{V}$ can be interpreted as being given by the ratio of the volume of the cylinder engendered by the collision cross section through the relative motion of both particles, $c_{r}\left(a^{\prime}, b^{\prime}\right)$, during $\Delta t$ to the volume of the cell:

$$
P_{\text {coll }}\left(a^{\prime}, b^{\prime}\right)=\frac{\tilde{\sigma}_{a b}\left(a^{\prime}, b^{\prime}\right) c_{r}\left(a^{\prime}, b^{\prime}\right) \Delta t}{V} .
$$

Collisions between particle pairs in a cell at a given time step are independent events, that is the probability of a collision occurring for a given $\left\{a^{\prime}, b^{\prime}\right\}$ pair is independent of those of all other pairs in the cell. Thus, the 
number of collisions that occurs during a time step is obtained by summing the probability of occurrence of each potential collision. When $a \neq b, N_{a} \times N_{b}$ collisions are susceptible to occur, so that

$$
N_{\text {coll }}(a, b)=\sum_{a^{\prime}=1}^{N_{a}} \sum_{b^{\prime}=1}^{N_{b}} P_{\text {coll }}\left(a^{\prime}, b^{\prime}\right)=\sum_{a^{\prime}=1}^{N_{a}} \sum_{b^{\prime}=1}^{N_{b}} \frac{\tilde{\sigma}_{a b}\left(a^{\prime}, b^{\prime}\right) c_{r}\left(a^{\prime}, b^{\prime}\right) \Delta t}{V} \quad a \neq b .
$$

and the average value of the cross section times the velocity amongst the $N_{a} \times N_{b}$ pairs is defined as:

$$
\overline{\tilde{\sigma}_{a b} c_{r}} \triangleq \frac{1}{N_{a} N_{b}} \sum_{a^{\prime}=1}^{N_{a}} \sum_{b^{\prime}=1}^{N_{b}} \tilde{\sigma}_{a b}\left(a^{\prime}, b^{\prime}\right) c_{r}\left(a^{\prime}, b^{\prime}\right) \quad a \neq b .
$$

In the case when collisions between particles of the same species are considered, that is when $a=b$, only $\left(\begin{array}{c}N_{a} \\ 2\end{array}\right)=\frac{N_{a}\left(N_{a}-1\right)}{2}$ collision pairs can take place so that Eqs. (19) and (20) respectively become

$$
N_{\text {coll }}(a, a)=\sum_{a^{\prime}=1}^{N_{a}} \sum_{b^{\prime}=a^{\prime}+1}^{N_{a}} P_{\text {coll }}\left(a^{\prime}, b^{\prime}\right)=\sum_{a^{\prime}=1}^{N_{a}} \sum_{b^{\prime}=a^{\prime}+1}^{N_{a}} \frac{\tilde{\sigma}_{a a}\left(a^{\prime}, b^{\prime}\right) c_{r}\left(a^{\prime}, b^{\prime}\right) \Delta t}{V} .
$$

and

$$
\overline{\tilde{\sigma}_{a a} c_{r}} \triangleq \frac{2}{N_{a}\left(N_{a}-1\right)} \sum_{a^{\prime}=1}^{N_{a}} \sum_{b^{\prime}=a^{\prime}+1}^{N_{a}} \tilde{\sigma}_{a a}\left(a^{\prime}, b^{\prime}\right) c_{r}\left(a^{\prime}, b^{\prime}\right)
$$

By using Eq. (17), Eqs. (19) and (21) can simultaneously be rewritten as:

$$
N_{\text {coll }}(a, b)=\frac{1}{1+\delta_{a b}} \frac{\overline{\tilde{\sigma}_{a b} c_{r}} \Delta t}{V} N_{a}\left(N_{b}-\delta_{a b}\right),
$$

which, using Eq. (17), becomes:

$$
N_{\text {coll }}(a, b)=\frac{N_{a} W_{p} W_{\text {rel }, b}\left(N_{b}-\delta_{a b}\right)}{\left(1+\delta_{a b}\right) V} \overline{\sigma_{a b} c_{r}} \Delta t .
$$

This gives an expression for the number of collisions that has to occur during $\Delta t$ amongst the particles of the numerical gas so that Eq. (16) is satisfied. To increase computational efficiency, the DSMC procedure considers potential collisions amongst a greater number of particle pairs, i.e. $\frac{\left(\sigma_{a b} c_{r}\right)_{\max }}{\bar{\sigma}_{a b} c_{r}} \geq 1$ times the $N_{\text {coll }}(a, b)$ number of collision pairs, denoted by $N_{\text {pairs }}(a, b)$ :

$$
N_{\text {pairs }}(a, b)=\frac{\left(\sigma_{a b} c_{r}\right)_{\max }}{\overline{\sigma_{a b} c_{r}}} N_{\text {coll }}(a, b),
$$

which, following Eq. (24), corresponds to

$$
N_{\text {pairs }}(a, b)=\frac{N_{a} W_{p} W_{\mathrm{rel}, b}\left(N_{b}-\delta_{a b}\right)}{\left(1+\delta_{a b}\right) V}\left(\sigma_{a b} c_{r}\right)_{\max } \Delta t .
$$

The collision probability between particles $a^{\prime}$ and $b^{\prime}$ of species $a$ and $b$ is suitably redefined from its expression in Eq. (18) to the following:

$$
P_{\text {coll }}\left(a^{\prime}, b^{\prime}\right)=\frac{\sigma_{a b}\left(a^{\prime}, b^{\prime}\right) c_{r}\left(a^{\prime}, b^{\prime}\right)}{\left(\sigma_{a b} c_{r}\right)_{\max }},
$$

so that Eq. (24) is indeed obtained on average over all collision pairs. In the case where $W_{\mathrm{rel}, a} \neq W_{\mathrm{rel}, b}$, the number of pairs to consider for collision, i.e. Eq. (26), has to be altered as the velocity of both particles cannot simultaneously change to conserve average momentum during the collision. This issue is addressed in the next two sections that culminate in the derivation of a form of Eq. (24), i.e. Eq. (43), that takes into account the collision dynamics of particles with different relative weights. 


\subsection{Collision Mechanics with relative weights}

In the absence of species relative weights and in the actual physical gas, the properties of both participating particles change after a collision. In the numerical gas, however, when particles with different relative weights participate in a collision, their properties cannot both equally be affected by it. If they were, the overall translational energy of particles and momentum would not be conserved on average in the gas and the effect of collisions on the species with the largest weight from that with the lowest would be systematically overestimated. In the following, we present the collision scheme that is most commonly used to handle collisions between particles with different relative weights which was first introduced in [17] p. 171. Because the scheme does not conserve energy, an energy-conserving collision scheme was subsequently proposed in [6]. The latter was for instance later used in [18] where a detailed description of the impact of relative weights on the modeling of chemical reactions via DSMC is given. An energy conserving scheme is, however, of limited utility for the test case that will later be studied as the residence time of particles inside the domain is relatively short with very few undergoing multiple collisions and is therefore not implemented. Non energy conservation is, however, much more important for closed systems, as discussed in [6], such as homogeneous heat baths where boundary conditions are not constantly resupplying new particles (and thus energy). Considering a collision between a particle of species $a$ with mass $m_{a}$ and relative weight $W_{\text {rel }, a}$ and one of species $b$ with mass $m_{b}$ and realtive weight $W_{\text {rel, } b}$, the pre-collision velocities of both particles are designated by $\vec{v}_{a}$ and $\vec{v}_{b}$ and their post-collision velocities are designated by $\vec{v}_{a}^{\prime}$ and $\vec{v}_{b}^{\prime}$. In the case where $W_{\text {rel, } a}=W_{\text {rel }, b}$, the post-collision velocities are denoted, respectively, by $\vec{v}_{a}^{*}$ and $\vec{v}_{b}^{*}$ and are obtained by the application of the DSMC collision operator $C_{\text {coll }}$ (i.e. the variable hard sphere model in present study) that is:

$$
\left(\vec{v}_{a}^{*}, \vec{v}_{b}^{*}\right)=C_{\text {coll }}\left(\vec{v}_{a}, \vec{v}_{b}\right)
$$

with

$$
\left\{\begin{array}{l}
\vec{v}_{a}^{\prime}=\vec{v}_{m}+\frac{m_{b}}{m_{a}+m_{b}} \vec{v}_{r}^{*} \\
\vec{v}_{b}^{\prime}=\vec{v}_{m}-\frac{m_{a}}{m_{a}+m_{b}} \vec{v}_{r}^{*}
\end{array}\right.
$$

where the center of mass velocity $\vec{v}_{m}$ is given by $\vec{v}_{m}=\frac{m_{a} \vec{v}_{a}+m_{b} \vec{v}_{b}}{m_{a}+m_{b}}$, while the expression for the post-collision relative velocity $\vec{v}_{r}^{*} \triangleq \vec{v}_{a}^{*}-\vec{v}_{b}^{*}$ as a function of $\vec{v}_{a}$ and $\vec{v}_{b}$ for the VHS (Variable hard sphere) model can be found in [2]. In order for the gas to conserve overall momentum during the collision, the following has to be satisfied:

$$
m_{a} W_{\mathrm{rel}, a} \vec{v}_{a}+m_{b} W_{\mathrm{rel}, b} \vec{v}_{b}=m_{a} W_{\mathrm{rel}, a} \vec{v}_{a}^{\prime}+m_{b} W_{\mathrm{rel}, b} \vec{v}_{b}^{\prime} .
$$

As $C_{\text {coll }}$ conserves momentum, $m_{a} \vec{v}_{a}+m_{b} \vec{v}_{b}=m_{a} \vec{v}_{a}^{*}+m_{b} \vec{v}_{b}^{*}$, so that Eq. (30) can be rewritten as:

$$
W_{\mathrm{rel}, b}\left(m_{a} \vec{v}_{b}^{*}+m_{b} \vec{v}_{b}^{*}\right)+\left(W_{\mathrm{rel}, a}-W_{\mathrm{rel}, b}\right) m_{a} \vec{v}_{a}=m_{a} W_{\mathrm{rel}, a} \vec{v}_{a}^{\prime}+m_{b} W_{\mathrm{rel}, b} \vec{v}_{b}^{\prime}
$$

Because we do not require conservation of energy, this equation is the only one that must be satisfied by the post collision velocities $\vec{v}_{a}^{\prime}$ and $\vec{v}_{b}^{\prime}$. They can thus be defined with a certain latitude. The most natural choice is to let the particle with the lowest relative weight be fully influenced by the collision, that is to assign it $\vec{v}^{*}$ as its post-collisional velocities. Using that assumption, Eq. (31) can readily be shown to admit the following two solutions:

$$
\left\{\begin{array}{l}
\vec{v}_{a}^{\prime}=\left(1-\frac{W_{\mathrm{rel}, b}}{W_{\mathrm{rel}, a}}\right) \vec{v}_{a}+\frac{W_{\mathrm{rel}, b}}{W_{\mathrm{rel}, a}} \vec{v}_{a}^{*} \\
\vec{v}_{b}^{\prime}=\vec{v}_{b}^{*}
\end{array}\right.
$$

$$
\left\{\begin{array}{l}
\vec{v}_{a}^{\prime}=\vec{v}_{a}^{*} \\
\vec{v}_{b}^{\prime}=\left(1-\frac{W_{\mathrm{rel}, a}}{W_{\mathrm{rel}, b}}\right) \vec{v}_{b}+\frac{W_{\mathrm{rel}, a}}{W_{\mathrm{rel}, b}} \vec{v}_{b}^{*}
\end{array}\right.
$$

The solution of either Eq. (32) or Eq. (33) are acceptable to conserve momentum during the collision. However, in order to properly simulate the effect of collisions, particles from the entire range of the velocity distribution function must be potentially allowed to exist in the flow. In particular, specifying velocities after each collision following Eq. (32) or (33), especially when $\frac{W_{\text {rel }, b}}{W_{\text {rel }, a}} \ll 1$ only produces a $\vec{v}_{a}^{\prime}$ or $\vec{v}_{b}^{\prime}$ that are very similar to their pre-collision values so that almost no "real" collision actually takes place in the flow. Hence, a solution to this, is to only satisfy Eq. (33) or (32) on average, that is in a probabilistic sense. This 
is done by allowing a collision to occur for the particles with the greatest relative weight with a probability given by the ratio of the weights, i.e.:

$$
\text { If } W_{\mathrm{rel}, a}<W_{\mathrm{rel}, b} \quad \vec{v}_{a}^{\prime}=\vec{v}_{a}^{*} \quad \vec{v}_{b}^{\prime}=\left\{\begin{array}{l}
\vec{v}_{b}^{*} \text { with } \mathrm{P}=\frac{W_{\mathrm{rel}, a}}{W_{\mathrm{rel}, b}} \\
\vec{v}_{b} \text { otherwise }
\end{array}\right.
$$

and

$$
\text { If } W_{\mathrm{rel}, a}>W_{\mathrm{rel}, b} \quad \vec{v}_{a}^{\prime}=\left\{\begin{array}{l}
\vec{v}_{a}^{*} \text { with } \mathrm{P}=\frac{W_{\mathrm{rel}, b}}{W_{\mathrm{rel}, a}} \\
\vec{v}_{a} \text { otherwise }
\end{array} \quad \vec{v}_{b}^{\prime}=\vec{v}_{b}^{*} .\right.
$$

We exclude the case where $W_{\mathrm{rel}, b}>W_{\mathrm{rel}, a}$ for Eq. (32) and the reverse for Eq. (33) to avoid generating large unphysical velocities after collision. As shown by Eqs. (34) and (35), a collision does not always result in a change of velocity for both particles when these have different relative weights. One must therefore define a real collision for a given particle as a collision in which its velocity is changed. The probability of such an event occurring during a collision of a particle from species $a$ with one from species $b$ is denoted by $P_{a b}$, while $P_{b a}$ refers to the probability that the particles from the $b$ species sees its velocity change in an encounter with one of $a$. Eqs. (34) and (35) respectively yield:

$$
W_{\mathrm{rel}, a}<W_{\mathrm{rel}, b} \quad P_{a b}=1 \quad P_{b a}=\frac{W_{\mathrm{rel}, a}}{W_{\mathrm{rel}, b}}
$$

and

$$
W_{\mathrm{rel}, a}>W_{\mathrm{rel}, b} \quad P_{a b}=\frac{W_{\mathrm{rel}, b}}{W_{\mathrm{rel}, a}} \quad P_{b a}=1 .
$$

so that one can remark that the identity $P_{a b}=\frac{W_{\mathrm{rel}, b}}{W_{\mathrm{rel}, a}} P_{b a}$ is always satisfied.

\subsection{Collision rates with relative weights}

When the mechanism detailed in the last section is used, a collision between two particles results in a real collision for one or both particles (as opposed to the case where both particles have the same relative weight, where a collision between two particles always results in a real collision for both). One must therefore distinguish between the real collision cross section that was previously introduced in Section 2.4, $\tilde{\sigma}_{a b}$, and the virtual collision cross section $\hat{\sigma}_{a b}$. The real collision cross section, $\tilde{\sigma}_{a b}$, is the cross section of the process " $\{$ Particle $a$ collides with particle $b$ AND its velocity is changed $\} "$ as opposed to $\hat{\sigma}_{a b}$ which is that of " $\{$ Particle $a$ collides with particle $b\} "$. Both are numerical cross sections (in that they are properties of the computational gas) and identical in the absence of relative weights. Because a collision of a particle of species $a$ with one of $b$ has a probability $P_{a b}$ of leading to a real collision:

$$
\tilde{\sigma}_{a b}=P_{a b} \hat{\sigma}_{a b}
$$

Hence using $\hat{\sigma}$, the single particle collision rate of Eq. (15) can be rewritten, using Eq. (17), as:

$$
\tilde{\nu}_{a b}^{(1)}=\tilde{n}_{b} \overline{\tilde{\sigma}_{a b} c_{r}}=P_{a b} \tilde{n}_{b} \overline{\hat{\sigma}_{a b} c_{r}}=n_{b} \overline{\sigma_{a b} c_{r}}
$$

so that using Eq. (14), the virtual collision cross section $\hat{\sigma}_{a b}$ is found to be:

$$
\hat{\sigma}_{a b}=\frac{W_{p} W_{\mathrm{rel}, b}}{P_{a b}} \sigma_{a b}
$$

By analogy with Eq. (23), which gives an expression for the number of real collisions that has to take place in a cell, the number of virtual numerical collisions in a cell is given by:

$$
\hat{N}_{\text {coll }}(a, b)=\frac{\overline{\hat{\sigma}_{a b} c_{r}} \Delta t}{V\left(1+\delta_{a b}\right)} N_{a}\left(N_{b}-\delta_{a b}\right) .
$$


which can be rewritten, using Eq. (40), as:

$$
\hat{N}_{\text {coll }}(a, b)=\frac{W_{p} W_{\mathrm{rel}, b} \overline{\sigma_{a b} c_{r}} \Delta t}{\left(1+\delta_{a b}\right) P_{a b} V} N_{a}\left(N_{b}-\delta_{a b}\right) .
$$

Following Eq. (25), the number of particle pairs to test for collision is thus

$$
\hat{N}_{\text {pairs }}(a, b)=\frac{W_{p} W_{\mathrm{rel}, b}\left(\sigma_{a b} c_{r}\right)_{\max } \Delta t}{\left(1+\delta_{a b}\right) P_{a b} V} N_{a}\left(N_{b}-\delta_{a b}\right) .
$$

The equation above corresponds to the "sophisticated DSMC" pair selection scheme introduced in [19], while the "no time counter" pair selection scheme [2] is obtained by replacing the $\left(N_{b}-\delta_{a b}\right)$ term by $\bar{N}_{b}$. Equation (43) however only gives an expression for the number of collision pairs between species $a$ and $b$ to consider from the standpoint of species $a$, so that the total number of collision pairs to test between species $a$ and $b, \hat{\mathrm{N}}_{\text {pairs }}\{a, b\}$, is given by:

$$
\hat{\mathrm{N}}_{\text {pairs }}\{a, b\}=\frac{1}{1+\delta_{a b}}\left[\hat{N}_{\text {pairs }}(a, b)+\hat{N}_{\text {pairs }}(b, a)\right] \text {, }
$$

noting that $\hat{\mathrm{N}}_{\text {pairs }}\{a, a\}=\hat{N}_{\text {pairs }}(a, a)$. Our implementation of the DSMC method with relative weights thus amounts to selecting the number of particle pairs indicated by Eqs. (44) and (43). Amongst those selected particle pairs, virtual collisions are assumed to occur with a probability of $P_{\text {coll }}$ given by Eq. (27), in which case they lead to the application of the collision mechanics described by Eq. (34) or (35). That is, a real collision always occurs for the particle with the lowest relative weight while a real collision only occurs with a probability given by the ratio of the relative weights for the other particle.

\section{Formulation of the adaptive procedure}

\subsection{Calculation of the time step}

\subsubsection{DSMC requirements}

The DSMC procedure is based on the decoupling of the two main mechanisms governing the dynamics of a rarefied gas: the ballistic motion of particles through physical space and their collisions. This basic assumption is an approximation as both are coupled in reality which in turn introduces a splitting error. In terms of the modeling, this means that collisions should really impact the "move" part of the procedure as they cannot be fully assumed to take place instantaneously at a fixed spatial location. An often cited guideline to mitigate this error is to ensure that the time step $\Delta t$ is less than the mean collision time $\bar{\tau}_{m}$. Because this splitting error most likely follows a power law, i.e. $(\Delta t)^{\eta}$ with $\eta>0$ [20], it can never be fully eliminated and is already significant for values of $\Delta t$ below $\bar{\tau}_{\mathrm{m}}$ as no distinct threshold phenomenon occurs when $\Delta t=\bar{\tau}_{\mathrm{m}}$. In the adaptive procedure, the value of the time step in each cell $i$ is thus set equal to a fraction of the mean collision time $\Xi_{\tau_{\mathrm{m}}}<1$ :

$$
\Delta t_{i}=\Xi_{\tau_{\mathrm{m}}} \bar{\tau}_{m, i}
$$

where $\bar{\tau}_{m, i}$ is defined according to Eq. (12). Particles should furthermore avoid crossing multiple cells during their movement. That is, they should not overfly a cell without having had a chance to collide with the particles occupying it. When this undesirable situation occurs, the move procedure prevents the proper modeling of collisions by spatially biasing the collision partner population of the particles being moved. An approximate measure of the average number of neighboring cells traversed by the particles contained inside cell $i$ during $\Delta t$ can be given by:

$$
K_{i}=\frac{\langle v\rangle_{i} \Delta t_{i}}{\langle\Delta x\rangle_{i}},
$$


where $\langle v\rangle_{i}$ designates the average velocity magnitude of the particles of cell $i$ and $\langle\Delta x\rangle_{i}$ designates a characteristic length of cell $i$ which can be taken as the value of its minimum edge length. We can prevent particles from crossing more than one cell on average during $\Delta t$ by imposing:

$$
K_{i} \leq \beta_{K, \max } \text { with } \beta_{K, \max }<1 .
$$

We note in passing that a value far below 1 is usually required for $\beta_{K \text {, max }}$ so that enough particles of the tail of the distribution function (which velocity magnitude greatly exceeds $\langle v\rangle_{i}$ ) do indeed not cross more than one cell per time step on average. Whereas Eqs. (45) and (47) provide upper bounds for the time step value, the latter should, however, also not be too small. This is because the autocorrelation spectrum $\rho\left(k^{\prime}, \Delta t\right)$ of sampled quantities as a function of the number of time steps $k^{\prime}$, for a given time step $\Delta t$ is such that $\rho\left(k^{\prime}, \Delta t\right)=\rho_{0}\left(k^{\prime} \Delta t_{0}\right)[20]$, so that reducing the time step increases the correlation between sampled quantities. This in turn reduces the rate of convergence of sampled quantities thereby requiring more sampling steps and hence making the simulation less computationally efficient. Without calculating the correlation between samples, this can for instance be accomplished by specifying a minimum bound for $K_{i}$ :

$$
\beta_{K, \min } \leq K_{i} \text { with } \beta_{K, \min }>0 .
$$

The two previous conditions, i.e. Eqs. (47) and (48) can thus be summarized as:

$$
\beta_{K, \min }<\frac{\langle v\rangle_{i} \Delta t_{i}}{\langle\Delta x\rangle_{i}}<\beta_{K, \max }
$$

which has to be satisfied for all the cells of the domain. The exact values of the $\beta_{K, \text { min }}$ and $\beta_{K \text {, max }}$ bounds must ultimately be determined by trial and error. Good results have been obtained for a variety of flows with $\beta_{K, \text { min }}=0.05$ and $\beta_{K, \max }=0.1$ which are the values that are used in the following. In the case when one species has a much higher velocity than the others or in the presence of a significant number of high speed particles from the tail of the velocity distribution function, the value of the upper bound $\beta_{K \text {,max }}$ must be further reduced. In such case, it may become preferable to either use the average velocity of the species with the greatest velocity instead of $\langle v\rangle_{i}$ or to base the bound on a fraction of the maximum particle velocity.

\subsubsection{Implementation considerations}

Applying the adaptive procedure essentially amounts to calculating $\left\{\Delta t_{i}^{k+1}\right\}_{i=1}^{N_{c}}$ at time step $t^{k}$ so that Eqs. (45) and (49) are satisfied and updating the simulation to account for the new time step. Because the adaptive procedures for the time step (and the weights) are applied during the march to steady state of the flow during which the latter greatly changes, it must carefully be applied so as not to excessively interfere with it. For that reason, as will be discussed in Section 3.4, the weights and time step are only updated every few thousands time steps to allow the flow to sufficiently develop between updates. The range of the time step is further restricted to prevent too low or high values from being used that would hinder the development of the flow:

$$
\Delta t_{\min }<\Delta t_{i}^{k+1}<\Delta t_{\max }
$$

The values of these two bounds obviously depend on that of the reference time step $\Delta t_{0}$. They should be, respectively, small and large enough so that the constraints of Eqs. (45) and (49) can be enforced. The magnitude of the increase or decrease of the time step is further restricted for each cell by imposing:

$$
\frac{1}{\Xi_{\Delta t, \max }} \leq \frac{\Delta t_{i}^{k+1}}{\Delta t_{i}^{k}} \leq \Xi_{\Delta t, \max } \text { with } \Xi_{\Delta t, \max }>1,
$$

which prevents possible oscillations in the overall number of particles due to a too tight coupling of the adaptive procedure with the flow. A moderate value should thus be used for $\Xi_{\Delta t \text {,max }}$ such as e.g. 2 .

We further desire a time step field that is relatively smooth, that is, without abrupt increases or decreases between adjacent cells. This is particularly necessary during the development period of the flow where large disparities between time steps are observed between zones with and without particles. Too sudden 
time step variations along the trajectory of particles furthermore degrades accuracy. A smooth time step field is obtained by performing a Laplacian smoothing, denoted by $L(\cdot)$, of the time step field $\Delta t^{k+1}$. After sequentially applying Eqs. (45), (49), (51) and (50) to obtain $\Delta t^{k+1}$, the smoothed time step field $\left\{\widehat{\Delta t}_{i}^{k+1}\right\}_{i=1}^{N_{c}}=L\left(\left\{\Delta t_{i}^{k+1}\right\}_{i=1}^{N_{c}}\right)$ is determined according to the following:

$$
L:= \begin{cases}\check{\Delta} t_{i}^{0} & =\Delta t_{i}^{k+1} \\ \check{\Delta} t_{i}^{l} & =\frac{1}{N_{b, i}+1}\left(\check{\Delta} t_{i}^{l-1}+\sum_{j=1}^{N_{b, i}} \check{\Delta} t_{J_{i, j}}^{l-1}\right) \text { with } 1 \leq l \leq N_{L} \\ \widehat{\Delta t}_{i}^{k+1} & =\check{\Delta} t_{i}^{N_{L}}\end{cases}
$$

The new average at step $l$ of the smoothing is obtained by averaging the value of the time step over the $N_{b, i}$ neighbors of cell $i, J_{i, j}$ designating the index of the cell that is the $j$ th neighbor of cell $i$. The averaging is repeated multiple times, in the course of which temporary time step fields $\left\{\check{\Delta} t_{i}^{l}\right\}_{i=1}^{N_{c}}$ are obtained, until a total of $N_{L}$ smoothing iterations have been performed.

The final step of the procedure is to suitably update the number of particles and the properties of the flow so that all flow quantities, when rescaled with the new time step value, are not altered. Because of the definition of number density following Eq. (3), the number of particles in cell $i$ of all $j$ species needs to be altered to keep the number density of the cell constant:

$$
N_{j, i}^{k+1}=N_{j, i}^{k} \frac{\Delta t_{i}^{k}}{\Delta t_{i}^{k+1}} \quad \forall j .
$$

When, $\frac{\Delta t_{i}^{k}}{\Delta t_{i}^{k+1}}>1$, the additional particles required are obtained by cloning the particles that are already present in the cell. The average number of particles in the cell, $\bar{N}_{j, i}^{k}$ (defined by Eq. (9)) is similarly updated:

$$
\bar{N}_{j, i}^{k+1}=\bar{N}_{j, i}^{k} \frac{\Delta t_{i}^{k}}{\Delta t_{i}^{k+1}} \quad \forall j,
$$

while no change is required for the average number of collisions between species $\bar{N}_{\text {coll }, i}^{k}(a, b)$. All the steps outlined above are briefly summarized below and the numerical values of all the parameters that are used in the test case of Section 4 are specified. The integration of the time step procedure within a DSMC code and its frequency of application is discussed in Section 3.4.

\section{Pseudo code of the time step update procedure: Update $\Delta t()$}

for all cells, $i=1, \ldots, N_{c}$, do:

i) Calculate $\tau_{m, i}$ with Eqs. (11) and (12) and enforce Eq. (45) with $\Xi_{\tau_{\mathrm{m}}}=0.2$.

ii) Calculate $K_{i}$ with Eq. (46) and enforce Eq. (49) with $\beta_{K, \min }=0.05$ and $\beta_{K, \max }=0.1$.

iii) Limit variation of $\Delta t_{i}^{k+1}$ with Eq. (51) using $\Xi_{\Delta t, \max }=2$.

iv) Check that $\Delta t_{i}^{k+1}$ is within the prescribed bounds of $\left[\Delta t_{\min }, \Delta t_{\max }\right]^{a}$.

iv) Smooth $\Delta t_{i}^{k+1}$ as in Eq. (52), using $N_{L}=20$ smoothing steps.

v) Clone/destroy particles as dictated by Eq. (53) and update $\bar{N}_{j, i}^{k}$ using Eq. (54). end

${ }^{a}$ Respectively small and large enough so that i) and ii) can be enforced.

\subsection{Calculation of cell weight}

\subsubsection{Motivation}

The accuracy of sampled quantities in a cell of a DSMC simulation depends on both the total number of particles in the simulation and the average number of particles in the cell. For fairly homogeneous test cases, such as channel flow, there is a direct connection between the average number of particles in a cell and 
the accuracy of sampled quantities so that the "20 particles per cell" [2] typical guideline may be used to guarantee accurate results. For more complex flow, such as axisymmetric jets, there is no direct relationship between the average number of particles per cell and the accuracy of sampled quantities [20], although an increase in the average number of particles in a cell is always associated with an increase in accuracy. Accurate results can, however, never be obtained in a cell with too few, e.g. $<5$ particles, so that one may ideally desire a minimum average number of particles in all cells of the domain as a necessary condition for accuracy.

Due to large number density gradients in the flow being simulated and/or large cell volume disparities in the mesh employed, DSMC users often employ spatially varying weights in their simulations to increase computational efficiency. This is in particular necessary for axisymmetric flows to obtain accurate results close to the centerline while avoiding too many particles in cells with greater volume further away from the centerline. Except for simple situations, such as single axisymmetric jets, the optimum shape of the distribution of weights to adopt is not obvious. Very often, users have to resort to a trial and error approach by running multiple simulations to determine a "good" weight distribution usually with the implicit goal of trying to obtain a constant average number of particles in all cells.

Building on the two previous ideas, the goal of the procedure presented in this section is thus to obtain a user-defined constant $N_{p \text {, want }}^{\text {tot }}$ particles per cell on average for all the cells of the domain. When $N_{\text {spec }}$ species are present in the flow and the adaptive procedure for the relative weights is used, such as in the following,

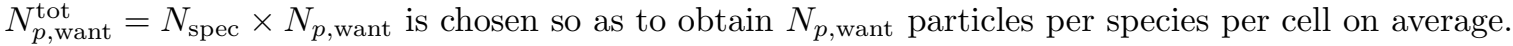

Having tested various implementations of the adaptive procedure, the best results have been obtained when the procedure for the relative weights is separated from that for the cell weight. This furthermore has the advantage of allowing the use of the procedure without relative weights. It is, however, possible to combine the procedures for the cell weight and the relative weights into a single one.

\subsubsection{Implementation}

The update equation for the value of the non-dimensional weight $\widetilde{W}_{p}$ (defined in Eq. (2)) is obtained by noting that the total average number of particles in cell $i, \bar{N}_{i}^{\text {tot, } k} \triangleq \sum_{j=1}^{N_{\text {spec }}} \bar{N}_{j, i}^{k}$ is proportional to $\widetilde{W}_{p, i}^{k}$, so that to have $\bar{N}_{i}^{\text {tot, } k+1}=N_{p \text {,want }}^{\text {tot }}$ at step $k+1$, we must impose:

$$
\widetilde{W}_{p, i}^{k+1}=\frac{\bar{N}_{i}^{\mathrm{tot}, k}}{N_{p, \text { want }}^{\text {tot }}} \widetilde{W}_{p, i}^{k}
$$

Similar conditions as for the time step are imposed, preventing too large increases or decreases while enforcing a lower and an upper bound with

$$
\frac{1}{\Xi_{\widetilde{W}_{p}, \max }} \leq \frac{\widetilde{W}_{p, i}^{k+1}}{\widetilde{W}_{p, i}^{k}} \leq \Xi_{\widetilde{W}_{p}, \max } \text { with } \Xi_{\widetilde{W}_{p}, \max }>1
$$

and

$$
\widetilde{W}_{p, \min }<\widetilde{W}_{p, i}^{k+1}<\widetilde{W}_{p, \max }
$$

Much like for the time step, it is important that the weight field be smooth in space and its variation gradual. This is to distribute the generation of cloned particles as equally as possible throughout space so as to promote the collisions of newly generated cloned particles with other particles. The aim of this is to $i$ ) Prevent the occurrence of the avalanche phenomenon which was discussed in Section 2.2.2 by breaking up packets of particles through collisions before they have the opportunity to form ii) Improve the contribution of newly generated cloned particles to the accuracy of the simulation by having them collide so as make them carry new information to the rest of the flow.

The new weight field is thus also smoothed with a Laplacian smoothing operator. The smoothing, however, has to take the axisymmetric geometry into account. This is because axisymmetric flows present very strong variations in the radial direction in which the cell volume varies as $\sim y$. Furthermore, because particles move parallel to the axis of symmetry in most axisymmetric flows, it is more important for the weight field to be 
smooth in the longitudinal direction than in the radial direction. The banded weight structures commonly used for axisymmetric jets, as in [2] p. 374, are, for instance, not smooth in the radial direction. In order to obtain accurate simulation results close to the centerline, enough particles have to be present in that region which necessitates, as the volume scales $\sim y$, that very low weights be employed for that region. Using centerline weights for the entire domain, however, leads to an excessive number of particles in the cells at greater radial distances, as $N \sim V \sim y$, which is the reason the weights of such cells are increased. Because the region requiring low weights close to the centerline is relatively limited in extent (i.e. it consists of only a few cells) compared to the zone requiring higher weights, the result of the application of the smoothing procedure described by Eq. (52) would be to increase the weights of the centerline region so as to make weight variations smoother in the radial direction. This would in turn systematically lead to an insufficient number of particles in the cells close to the centerline. Possible solutions to the issue include using radial weights in the smoothing, so that $\left\{w_{i} \widetilde{W}_{p, i}\right\}_{i=1}^{N_{c}}$ is smoothed with for instance $w_{i}=\frac{1}{y_{c, i}}$ or the solution that is adopted in this work, which consists in excluding centerline cells from the Laplacian smoothing thus forcing other neighboring cells to conform to their lower weight values. The smoothed weight field $\widehat{\widetilde{W}}_{p}$ is obtained by applying smoothing operator $L_{\text {axi }}$, i.e. $\left\{\widehat{\widetilde{W}}_{p, i}^{k+1}\right\}_{i=1}^{N_{c}}=L_{\text {axi }}\left(\left\{\widetilde{W}_{p, i}^{k+1}\right\}_{i=1}^{N_{c}}\right)$, which we define as follows:

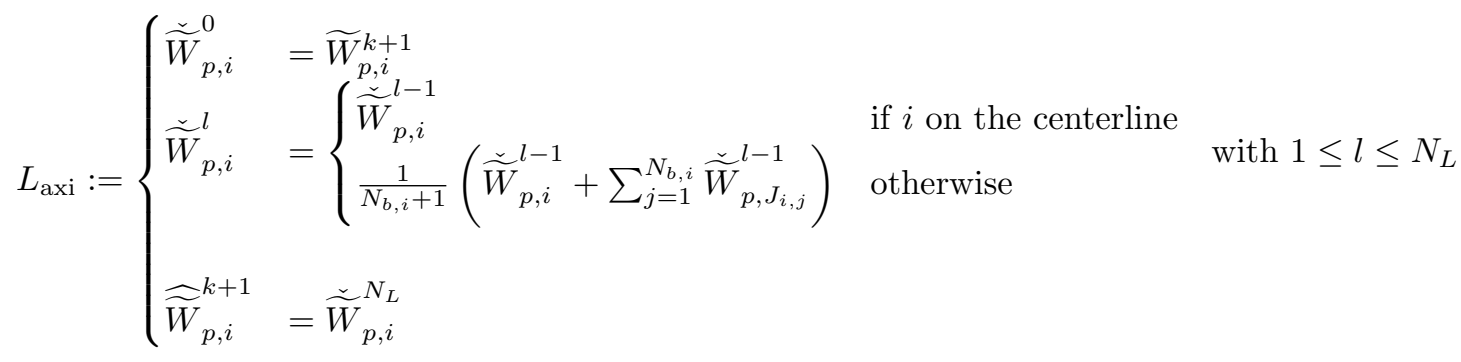

This smoothing operator increases the average number of particles in cells that are not on the centerline, but in close proximity to it, past $N_{p \text {,want }}$ which, however, represents a small computational penalty (in view of the increased accuracy afforded by the scheme). The adaptive weight procedure concludes by an update of the number of particles in each cell so that each maintains the same number density that it had with the old weight value:

$$
N_{j, i}^{k+1}=N_{j, i}^{k} \frac{\widetilde{W}_{p, i}^{k}}{\widetilde{W}_{p, i}^{k+1}} \quad \forall j
$$

while the average numbers of particles and collisions are suitably updated following

$$
\bar{N}_{j, i}^{k+1}=\bar{N}_{j, i}^{k} \frac{\widetilde{W}_{p, i}^{k}}{\widetilde{W}_{p, i}^{k+1}} \quad \forall j
$$

and

$$
\bar{N}_{\mathrm{coll}, i}^{k+1}(a, b)=\bar{N}_{\mathrm{coll}, i}^{k}(a, b) \frac{\widetilde{W}_{p, i}^{k}}{\widetilde{W}_{p, i}^{k+1}} \quad \forall a, \forall b .
$$

All the different steps of the weight update algorithm are summarized in the pseudo code below. 


\section{Pseudo code of the $\widetilde{W}_{p}$ update procedure: $\operatorname{Update} W_{p}()$}

for all cells, $i=1, \ldots, N_{c}$, do:

i) Calculate the new weight value $\widetilde{W}_{p, i}^{k+1}$ with Eq. (55)

iii) Limit variation of $\widetilde{W}_{p, i}^{k+1}$ with Eq. (56) using $\Xi_{\widetilde{W}_{p}, \max }=2$.

iv) Check that $\widetilde{W}_{p, i}^{k+1}$ is within the prescribed bounds of $\left[\widetilde{W}_{p, \min }, \widetilde{W}_{p, \max }\right]$ a

iv) Smooth $\widetilde{W}_{p, i}^{k+1}$ as in Eq. (58), using $N_{L}=20$ smoothing steps.

v) Clone/destroy particles as dictated by Eq. (59) and update averages following Eqs. (60) and (61). end

${ }^{a}$ The value of the bounds depend on that of $W_{p, 0}$ so are not specified here.

\subsection{Calculation of relative weights}

The aim of the adaptive procedure for the relative weights is to determine $N_{\text {spec }}$ different relative weight fields, $\left\{W_{\text {rel }, j, i}\right\}_{j=1}^{N_{\text {spec }}}$ for all $i$ cells, such that all contain $N_{p \text {, want }}$ particles of species $j$. It essentially consists in applying the procedure that was previously presented for the cell weight to each individual $j$ species. Due to the similarity of this procedure with that for the cell weights, it will only be presented in the form of the pseudo-code shown below.

\section{Pseudo code of the update procedure for the relative weights: $U p d a t e W_{\text {rel }}()$}

for all cells, $i=1, \ldots, N_{c}$, do:

for all species, $j=1, \ldots, N_{\text {spec }}$, do:

i) Calculate the new relative weight value:

$$
W_{\mathrm{rel}, j, i}^{k+1}=\frac{\bar{N}_{j, i}^{k}}{N_{p, \text { want }}} W_{\mathrm{rel}, j, i}^{k}
$$

ii) Limit variation of $W_{\text {rel,j,i}}^{k+1}$ with Eq. (56) using $\Xi_{\widetilde{W}_{p}, \max }=2$.

iii) Check that $W_{\mathrm{rel}, j, i}^{k+1}$ is within the prescribed bounds of $\left[W_{\mathrm{rel}, j, \min }, 1\right]^{a}$

iv) Check that $\widetilde{W}_{p} \times W_{\mathrm{rel}, j, i}^{k+1}$ is within the prescribed bounds of $\left[\left(\widetilde{W}_{p} W_{\mathrm{rel}, j}\right)_{\min }, \widetilde{W}_{p, \max }\right]^{b}$

v) Smooth $W_{\mathrm{rel}, j, i}^{k+1}$ as in Eq. (52), using $N_{L}=20$ smoothing steps.

vi) Clone/destroy particles following

$$
N_{j, i}^{k+1}=N_{j, i}^{k} \frac{W_{\mathrm{rel}, j, i}^{k}}{W_{\mathrm{rel}, j, i}^{k+1}}
$$

vii) Update the average numbers of particles with Eq. (60) using the relative weight ratio and the average number of collisions between species as follows:

for all species, $j^{\prime}=1, \ldots, N_{\text {spec }}$, do

$$
\bar{N}_{\mathrm{coll}, i}^{k+1}\left(j, j^{\prime}\right)=\bar{N}_{\mathrm{coll}, i}^{k}\left(j, j^{\prime}\right) \frac{W_{\mathrm{rel}, j, i}^{k}}{W_{\mathrm{rel}, j, i}^{k+1}} \text { and } \bar{N}_{\mathrm{coll}, i}^{k+1}\left(j^{\prime}, j\right)=\bar{N}_{\mathrm{coll}, i}^{k}\left(j^{\prime}, j\right) \frac{W_{\mathrm{rel}, j, i}^{k+1}}{W_{\mathrm{rel}, j, i}^{k}}
$$

end

end

end

${ }^{a}$ The bounds are test case dependent although we always require that relative weights be less than 1 so that $W_{\text {rel, } j, \max }=1$. ${ }^{b}$ Ibid. 


\subsection{Integration in a DSMC code}

The time step and weights have to be updated multiple times as the flow develops from its initial conditions at time step $k=0$ to its steady state. This is because of the coupling mechanism that exists between the weight and time step values and the flow. The particle velocities and locations depend on the time step and weight values while these both depend on all the particles. The flow must further be allowed to sufficiently develop between each time step or weight change so that these in turn do not excessively impact the flow. Following these considerations, the solution that is retained is thus to alternatively apply every $N_{\text {adapt }}$ steps either simultaneously the adaptive procedure for the time step and cell weight or the procedure for the relative weights. The adaptive procedure is applied until the flow reaches steady state, i.e. $k=N_{\text {stop }}$ at which point the sampling of flow quantities commences. The context and location of the adaptive procedures within a standard DSMC implementation is shown in the following pseudo-code:

\section{Integration of the adaptive procedure within a DSMC code:}

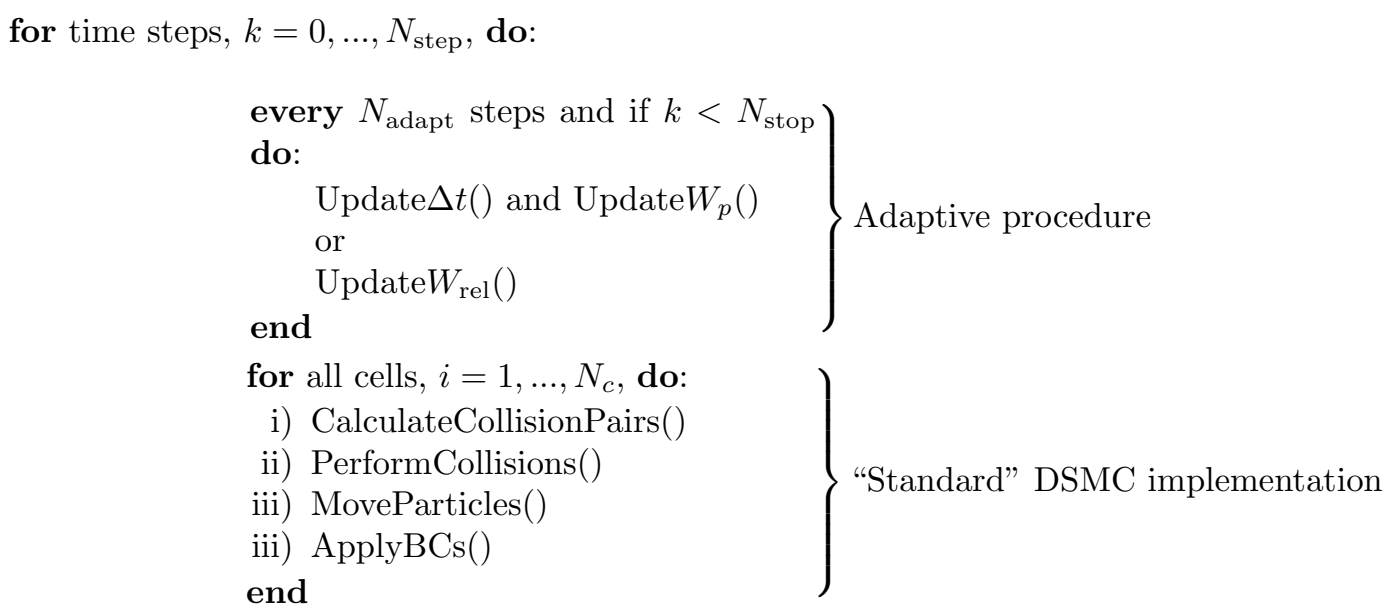

end

The application of the procedure creates a sharp rise or drop in the total number of particles in the domain as particles are either created or destroyed to conform to the new time step and weight fields following Eqs. (53), (59) and (63). The magnitude of these discontinuities in the number of particles gradually decays as the flow and its weight and time fields approach steady state. A typical evolution of the number of particles produced by the adaptive procedure is shown in Fig. 1 for the test cases summarized in Table 2. The $\{$ weight+time step $\}$ procedure is applied every odd thousand of steps while that for the relative weights is applied every even thousand of steps, thus corresponding to $N_{\text {adapt }}=1,000$ in the pseudo code above. For the following test case, the application of the adaptive procedure is stopped at $N_{\text {stop }}=100,000$ at which point the sampling of flow quantities begins. An estimate of the state of convergence of the time step and weight fields produced by the adaptive method can be obtained by monitoring the relative change in the number of particles when the method is applied. When the change is sufficiently small, the adaptive method can be assumed to have converged and the flow to be in steady state. Sampled quantities can, however, also be suitably rescaled so as to allow for sampling during the application of the adaptive procedure which we have chosen not to do in the following. It is furthermore not necessary to wait for the adaptive method to have fully converged to the final weight and time step field before stopping its application. Significant efficiency and accuracy benefits can already be obtained before full convergence such as at $k=60,000$ e.g. in Fig. 1. As the adaptive procedure is relatively independent of the main DSMC algorithm, as shown by the pseudo code above, it is fairly easy to implement in an existing DSMC code or in a stand-alone code that would process DSMC simulation result files. The computational cost of the procedure compared to that of the DSMC simulation is negligible as it is seldom applied, e.g. every 1,000 steps here, and only $\mathcal{O}\left(N_{\text {particles }}\right)$ at its worst (if every particle is cloned). The adaptive method is furthermore straightforwardly 
parallelizable. If the cells of the DSMC simulation are distributed amongst multiple processors, the only part of the procedure that requires a sharing of data between processors is the smoothing operation.

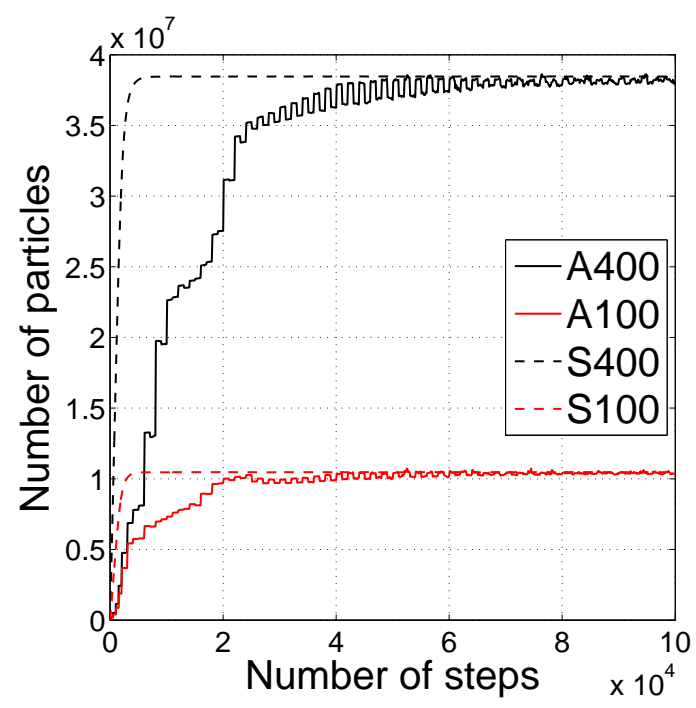

Figure 1: Evolution of the number of particles in the simulations from an empty domain at step 0 to steady state with $N_{\text {adapt }}=1,000$ for the test cases summarized in Table 2 .

\section{Application of the adaptive method}

In order to illustrate the increased computational efficiency brought about by the use of the adaptive method, it is applied to a test case consisting of 3 species which is presented in the next section. Results of the application of the method are shown and the efficiency of the method is then evaluated by comparing it against a reference solution.

\subsection{Test Case}

The test case depicted in Fig. 2 is $2 \mathrm{D}$ axisymmetric and consists of two jets of the same $D=0.002 \mathrm{~m}$ diameter separated by distance $\Delta L=0.25 \mathrm{~m}$. Jet 1 emits species 1 and 3 while jet 2 emits species 2 for which respective boundary conditions are summarized in Table 1 . This test case was chosen due to the difficulties that a traditional DSMC approach has to solve such flows. Because of spatial diffusion and the effects of collisions, it is indeed challenging to obtain accurate sampled quantities for a species in front of the 
jet opposite that from which it is emitted. Good resolution in such a zone is for instance required in the case of chemically reacting flows such as that presented in [21]. All species are assumed to be in equilibrium with no bulk velocity in the $y$ or $z$ directions when emitted. The Knudsen number of the two jets, based on their radius and VHS equilibrium results is 0.015 and all species are emitted at Mach number of 1 . All species are assumed to consist of argon and no chemical reactions take place so that the tag, i.e. the species number of a particle, remains constant. Supersonic outflow conditions are used for the left, top and right boundaries while a de facto axisymmetric boundary condition is enforced by the axisymmetric move procedure at the centerline (see [2] p. 370). Specular reflection wall boundary conditions are imposed at the wall between jet 2 and the outflow of the domain. Collisions between particles are modeled via the VHS model [2] with a reference diameter of $4.17 \times 10^{-10} \mathrm{~m}$ at $273 \mathrm{~K}$ and a temperature exponent of 0.81 . The sophisticated DSMC pair selection scheme of Eq. (43) is used while further details concerning our implementation of the DSMC method can be found in [22]. The mesh consists of 60,200 triangular cells with a maximum value for $\frac{\langle\Delta x\rangle}{\lambda}$ of 0.40 close to the inlet of the two jets.

\begin{tabular}{|l|l|c|c|}
\hline & Number density $\mathrm{m}^{-3}$ & $x$-Bulk velocity $\mathrm{m} / \mathrm{s}$ & Temperature $[\mathrm{K}]$ \\
\hline Jet 1 & & 589 & \\
1 & $1.2574 \times 10^{23}$ & 589 & 1,000 \\
3 & $1.2701 \times 10^{21}$ & & 1,000 \\
\hline \hline Jet 2 & & -589 & 1,000 \\
2 & $1.2701 \times 10^{23}$ & & \\
\hline
\end{tabular}

Table 1: Jet boundary conditions.

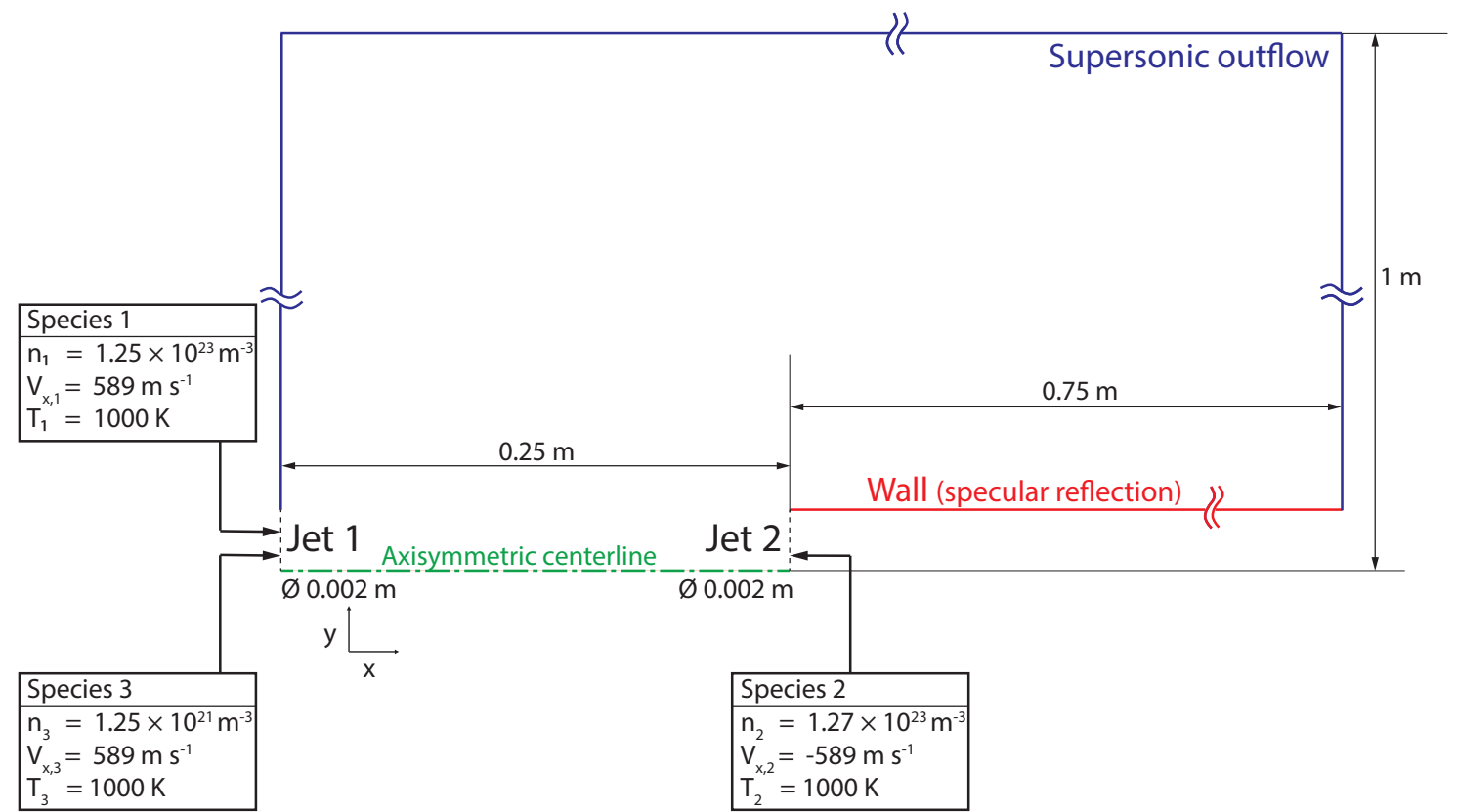

Figure 2: Schematic of test case with boundary conditions and dimensions. 


\subsection{Results}

The results presented in the following are obtained by using the numerical parameters indicated in Tables 2 and 3. The value of the bounds used in the application of the adaptive procedure are reported between brackets along with the values attained by the corresponding variable in the actual simulation (between parenthesis). For instance in the case of simulation A400, the minimum allowable value for the relative weight of all species is set at $10^{-8}$, while the minimum relative weight value obtained by the adaptive procedure for species 1 is $30 \times 10^{8}$, i.e. greater than the minimum bound. Test case A400 represents an attempt with a relatively moderate particle count to resolve the flow at hand with the help of the adaptive method for which detailed results will be presented in the following. More accurate results can be obtained by increasing the main governing parameter of the method, i.e. $N_{p \text {, want }}$, which will later be accomplished with test case A4800 to obtain a reference "exact" solution. Conversely, the computational cost of the simulation can be lessened, at the cost of reduced accuracy, by decreasing $N_{p \text {, want }}$ as done with test case A100. The adaptive procedure is applied from time step $k=0$ (where the computational domains contains no particles) up until time step 100,000 at which point the sampling of flow quantities begins. The evolution of the total number of particles in the domain for the period of time during which the adaptive procedure is applied is shown in Fig. 1. The normalized weight $\widehat{W}_{p}=\widetilde{W}_{p} \widetilde{\Delta} t$ and time step $\widetilde{\Delta} t$ obtained with the method for test case A400 are shown in Fig. 4. A relatively low time step value is obtained close to the inlets of the two jets where the large number density translates into a low mean collision time which is shown in the bottom part of Fig. 5. This in turn constrains the value of the time step following Eq. (45). To combat an excessive increase in the number of particles in the radial direction due to the radial growth of cell volumes, visible in Fig. 5, the radially increasing weight field shown in Fig. 4 is obtained. It is somewhat similar to the banded patterns, i.e. $\widetilde{W}_{p} \sim y$, commonly used to simulate many axisymmetric flows. Figure 6 displays the relative weight fields obtained for species 1 and 2. As expected, the relative weight of a given species gradually decreases away from the jet from which it is emitted, ultimately reaching its minimum value in front of the opposing jet. The time step and weight fields generated by the procedure are indeed seen to produce an almost constant $N_{p \text {,want }}$ average number of particles throughout the domain as observed in Fig. 7 which shows that $\bar{N}_{1} \sim \bar{N}_{2} \sim 400$, as would be expected, in the A400 case.

\begin{tabular}{|c|c|c|c|c|}
\hline Designation & A400 & A100 & S400 & S100 \\
\hline$W_{p, 0}$ & $5.6 \times 10^{12}$ & $5.6 \times 10^{12}$ & $0.71 \times 10^{12}$ & $2.42 \times 10^{12}$ \\
\hline$\Delta t_{0}[\mathrm{~s}]$ & $5.0 \times 10^{-8}$ & $5.0 \times 10^{-8}$ & $5.0 \times 10^{-8}$ & $5.0 \times 10^{-8}$ \\
\hline Adaptive $\widetilde{\Delta} t$ & YES & YES & $\mathrm{NO}, \widetilde{\Delta} t$ from $\mathrm{A} 400$ & $\mathrm{NO}, \widetilde{\Delta} t$ from $\mathrm{A} 100$ \\
\hline Adaptive $W_{p}$ & YES & YES & $\mathrm{NO}, W_{p}$ from $\mathrm{A} 400$ & $\mathrm{NO}, W_{p}$ from $\mathrm{A} 400$ \\
\hline Adaptive $W_{\text {rel }}$ & YES & YES & NO & NO \\
\hline$N_{p, \text { want }, 1,2,3}$ & 400 & 100 & $\mathrm{~N} / \mathrm{A}$ & $\mathrm{N} / \mathrm{A}$ \\
\hline $\begin{array}{l}\text { Total realized number } \\
\text { of particles }(M)\end{array}$ & 38.6 & 10.5 & 38.6 & 10.5 \\
\hline $\begin{array}{l}\text { Realized number } \\
\text { of particles for } \\
\text { species }(1,2,3)(\mathrm{M})\end{array}$ & $(12.9,13.2,12.3)$ & $(3.7,3.7,3.1)$ & $(18.6,19.7,0.19)$ & $(5.1,5.3,0.05)$ \\
\hline$\widetilde{\Delta}_{t, \min }, \widetilde{\Delta}_{t, \max }$ & {$\left[10^{-12}, 10^{12}\right]\left(3.310^{-3}, 109.7\right)$} & {$\left[10^{-12}, 10^{12}\right]\left(6.610^{-3}, 116.7\right)$} & $\left(3.310^{-3}, 109.7\right)$ & $\left(6.610^{-3}, 116.7\right)$ \\
\hline$\widetilde{W}_{p, \min }, \widetilde{W}_{p, \max }$ & {$\left[2^{-10}, 2^{50}\right]\left(1.910^{-3}, 1\right)$} & {$\left[2^{-10}, 2^{50}\right]\left(1.910^{-3}, 1\right)$} & $\left(1.910^{-3}, 1\right)$ & $\left(1.910^{-3}, 1\right)$ \\
\hline$W_{\text {rel }, \min , 1,2,3}$ & {$\left[10^{-8}\right],(30,1.05,1) 10^{-8}$} & {$\left[10^{-8}\right],(34,1.2,1) 10^{-8}$} & 1 & 1 \\
\hline$W_{\mathrm{rel}, \max , 1,2,3}$ & $(0.42,0.87,0.004)$ & $(1,1,0.017)$ & 1 & 1 \\
\hline$\left\{\widetilde{W}_{p} W_{\text {rel }}\right\}_{\min , 1,2,3}$ & {$\left[10^{-8}\right],(2.0,2.0,1.8) 10^{-9}$} & {$\left[10^{-8}\right],(2.0,2.0,1.8) 10^{-9}$} & $(2.0,2.0,1.8) 10^{-9}$ & $(2.0,2.0,1.8) 10^{-9}$ \\
\hline
\end{tabular}

Table 2: Parameters of different test cases presented. The set values of the bounds are shown between brackets while actually observed values are reported between parenthesis. 


\begin{tabular}{|c|c|c|}
\hline Designation & A4800 & A100-1em10 \\
\hline$W_{p, 0}$ & $5.6 \times 10^{12}$ & $5.6 \times 10^{12}$ \\
\hline$\Delta t_{0}[\mathrm{~s}]$ & $5.0 \times 10^{-8}$ & $5.0 \times 10^{-8}$ \\
\hline Adaptive $\widetilde{\Delta} t$ & YES & YES \\
\hline Adaptive $\widetilde{W}_{p}$ & YES & YES \\
\hline Adaptive $W_{\text {rel }}$ & YES & YES \\
\hline$N_{p, \text { want }, 1,2,3}$ & 4800 & 100 \\
\hline $\begin{array}{l}\text { Realized number } \\
\text { of particles }(\mathrm{M})\end{array}$ & 487 & $\times($ Not run to steady state $)$ \\
\hline $\begin{array}{l}\text { Realized number } \\
\text { of particles for } \\
\text { species }(1,2,3)(\mathrm{M})\end{array}$ & $(161,174,153)$ & $\times$ \\
\hline$\widetilde{\Delta}_{t, \min }, \widetilde{\Delta}_{t, \max }$ & {$\left[10^{-12}, 10^{12}\right]\left(8.810^{-4}, 105.4\right)$} & {$\left[10^{-12}, 10^{12}\right]\left(11.110^{-4}, 126.5\right)$} \\
\hline$\widetilde{W}_{p, \min }, \widetilde{W}_{p, \max }$ & {$\left[2^{-10}, 2^{50}\right]\left(1.910^{-3}, 1\right)$} & {$\left[2^{-10}, 2^{50}\right]\left(1.910^{-3}, 1\right)$} \\
\hline$W_{\mathrm{rel}, \min , 1,2,3}$ & {$\left[10^{-10}\right],(23.3,1.01,1.09) 10^{-10}$} & {$\left[10^{-10}\right],(1.19,1.19,1.19) 10^{-7}$} \\
\hline$W_{\mathrm{rel}, \max , 1,2,3}$ & {$[1],(0.089,0.087,0.0009)$} & {$[1],(1,1,1)$} \\
\hline$\left\{\widetilde{W}_{p} W_{\text {rel }}\right\}_{\min , 1,2,3}$ & {$\left[10^{-11}\right],(2.61,2.39,1.66) 10^{-11}$} & {$\left[10^{-10}\right],(2.33,2.33,2.33) 10^{-10}$} \\
\hline
\end{tabular}

Table 3: Parameters of different test cases presented (Continued). The set values of the bounds are shown between brackets while actually observed values are reported between parenthesis.

\subsection{Conditions of use and limitations of the scheme}

The explicitly stated goal of the adaptive procedure, i.e. to obtain $N_{p \text {,want }}$ particles per species in all cells of the domain, c.f. Eqs. (55) and (62), although certainly possible from a purely numerical standpoint, reveals itself to be somewhat shortsighted as far as accuracy is considered once the scheme is actually implemented. This is in large part due to the occurrence of the avalanching phenomenon that was described in Section 2.2.2 and in the Appendix, i.e. the fact that one cannot decrease weights ad infinitum in a certain direction to offset the natural decrease in particle count brought about by a negative number density gradient if the flow is not collisional enough. The avalanching phenomenon is only a very obvious manifestation of a broader phenomenon, i.e. the fact that cloning particles at a weight interface does not introduce new information in the simulation. This explains why the expected level of accuracy that one should expect of a given cell in a simulation with spatially varying weights that contains $\bar{N}$ particles on average is inferior to what is obtained when such a cell contains the same average number of particles in a simulation where uniform weights are employed. The decrease in the quality of the information content of a particle in a simulation with spatially varying weights is, however, extremely difficult to quantify a priori, that is before actually conducting a simulation. We also note than an alternate treatment of particle cloning at weight interfaces (such as that mentioned in Section 2.1.2) might, in principle, be able to improve this situation by promoting the collision of cloned particles. In view of the additional complications introduced by such method, it was however not utilized in the current study. During the course of the development and testing of the adaptive procedure, two important best practices have been identified. 1) The average number of particles per cell that one is aiming for, i.e. $N_{p \text {,want }}$, should be relatively high bearing in mind that the commonly cited guideline of " 20 particles per cell for accuracy" does not apply when cloned particles are present. This is the reason why the results that were previously shown, i.e. A400 and A100 are obtained with, respectively, 400 and 100 particles, far in excess of what one traditionally aims for in a DSMC simulation. 2) One should not use minimum

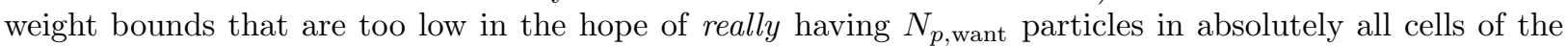
domain. The value of the bounds should be commensurate with that of $N_{p \text {,want }}$ so that the avalanching phenomenon does not become too manifest. When the occurrence of such a phenomenon becomes apparent, it is the sign that sampled quantities will be negatively impacted by the phenomenon in zones where they occur and that at least equally good results are obtainable without the use of such low weights for the same $N_{p \text {,want }}$ value. This is the reason behind the use of relatively high minimum weights bounds, i.e. $10^{-8}$ in Table 2, for test cases A100 and A400. Looking at the sampled value of the number density in Fig. 3, there is an approximate 12 order of magnitude drop (from $\sim 10^{23}$ to $\sim 10^{11}\left[\mathrm{~m}^{-3}\right]$ ) between the value of the number density at the outlet of jet and that of jet 2, although the latter cannot be properly resolved, even 

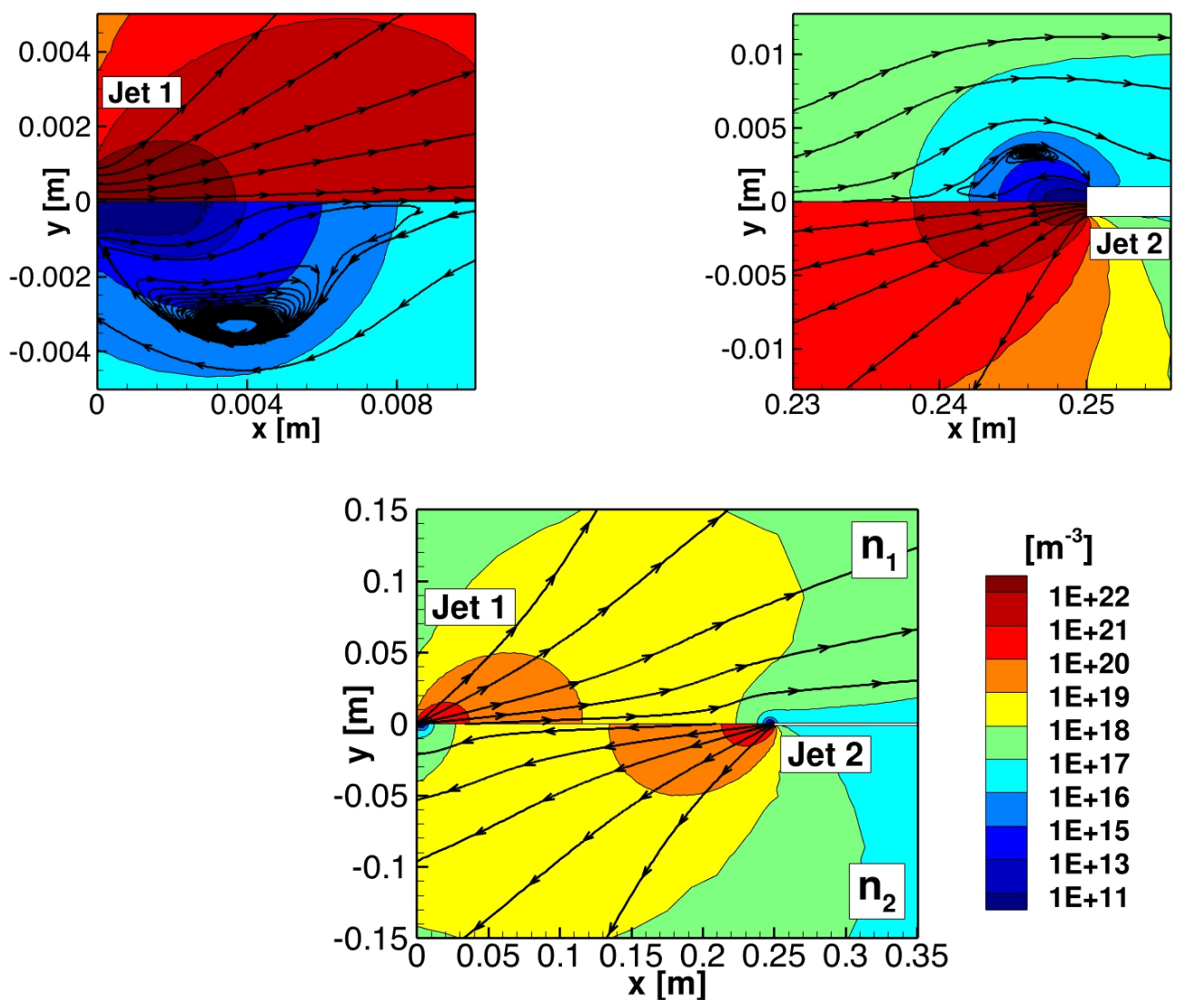

Figure 3: Sampled number density of species 1 (top) and 2 (bottom). Streamlines calculated with the sampled velocity of species 1 (top) and 2 (bottom) emitted respectively by jets 1 and 2 are shown.

with the adaptive procedure. As the mesh geometry is identical close to the two inlets so that cells have the same volume in front of the two jets (Fig 5), this would in turn entail that a relative weight of at most $\frac{1}{10^{12}}=10^{-12}$ is needed to properly populate that region with the species emitted by the opposite jet. This is far below the specified weight of $10^{-8}$, which explains the very low average number of particles of species 1 observed close to jet 2 in Fig. 7. One can, for instance, try to populate the region next to jet 2 with more particles of species 1 by lowering the minimum allowable $W_{\text {rel,1 }}$ from the $10^{-8}$ value that is used for test case A100, to $10^{-10}$, as done in test case A100-1em10 (outlined in Table 3). The results of the latter test case are presented in Figs. 8 and 9 where the hallmarks of the avalanching phenomenon are clearly visible. One sees in Fig. 8 groups of large numbers $\left(\sim 10^{5}\right)$ of particles of species 1 approaching jet 2 with $V_{x, 1}>0$, as shown in Fig. 9. Particles of species 1 are seen emanating from that packet of particles with $V_{x, 1}<0$ velocities due to collisions with particles of species 2 which become increasingly prevalent as it approaches the outlet of jet 2. Those particles form the "tail" of the packet of particles that is clearly visible in light blue in Fig. 8 and dark blue in Fig. 9. The infrequent occurrence of groups containing large numbers of particles of species 1 is the main mechanism populating the region close to the inlet of jet 2 with particles of species 1 . The collisions of these particles with those of species 2 is what gives rise to the negative value of the sampled $V_{x, 1}$ in that region which is shown in Fig. 9. In fluid dynamics terms, this corresponds to the occurrence of a recirculation region which is clearly visible when looking at the streamlines in Fig. 3 . The deleterious effects of the avalanching phenomenon on sampled quantities is clearly shown in Fig. 9 where streaks are visible in the sampled $V_{x, 1}$ contours. One further computational consequence of the avalanching phenomenon, visible in Fig. 8, is that some cells are seen to contain very large numbers of particles, i.e. in 

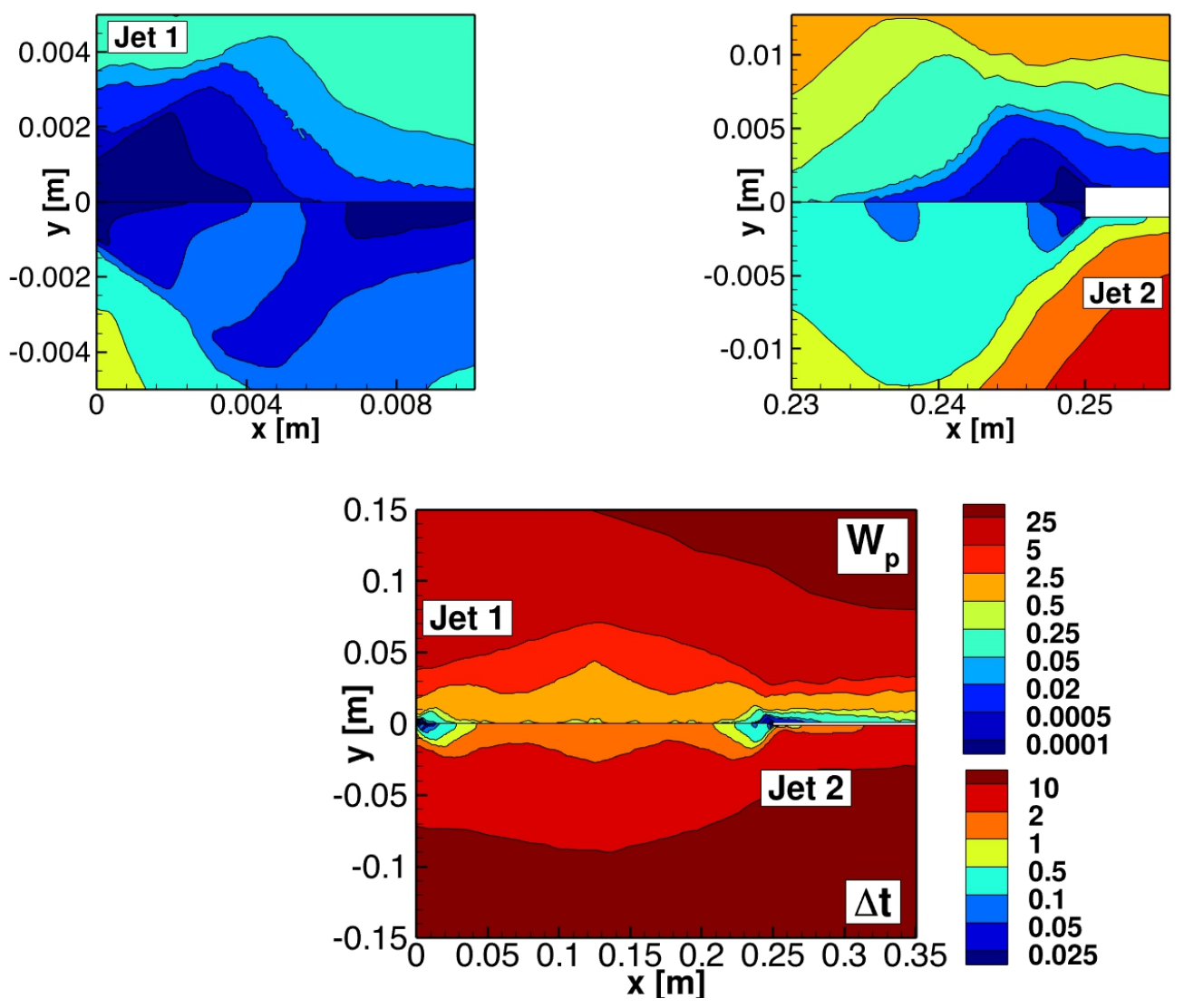

Figure 4: Normalized cell weight $\widehat{W}_{p}=\widetilde{W}_{p} \widetilde{\Delta} t$ and time step $\widetilde{\Delta} t$ (bottom) for A400.

excess of 200,000 in the present case. Because they only seldom do, this only translates into a moderate increase in the average number of particles, calculated with Eq. (9), as seen in the lower part of Fig. 8 which in turn does not lead to the adaptive procedure increasing the value of the weights in that region. Most DSMC codes, such as that used here, can, however, not contain an arbitrarily large number of particles without ultimately running out of memory, so that the minimum bound value employed has to be limited. If for instance, as previously described, one uses $W_{\text {rel, }, 1}=10^{-12}$ in the zone in front of jet 2 , whenever a particle of species 1 ultimately makes its way unhindered (i.e. without collisions) from jet 1 to jet 2 , and is cloned from $\widetilde{W}_{p} W_{\text {rel, } 1} \sim 1$ to $10^{-12}$, one ultimately obtains at some time step $\sim 10^{12}$ particles of species 1 distributed amongst a few cells in front of jet 2 similarly to what is visible in the upper part of Fig. 8 around the tip of the packet of particles. This potential memory issue is one further reason that cautions against the use of excessively low weights.

\subsection{Accuracy and Efficiency}

As previously stated, most DSMC simulations of axisymmetric flows are at least conducted with radially varying weights [2] [8] [9] with values that increase with the distance to the centerline. This is performed to increase particle counts close to the centerline while preventing an excessive number of particles in cells further removed from it as their volume scales with $y$. Whenever such capability exists [10] [11], a spatially varying time step is also used in DSMC simulations where large number density (and thus mean collision time) gradients exist. This prevents the value of the overall time step of the simulation from being dictated 

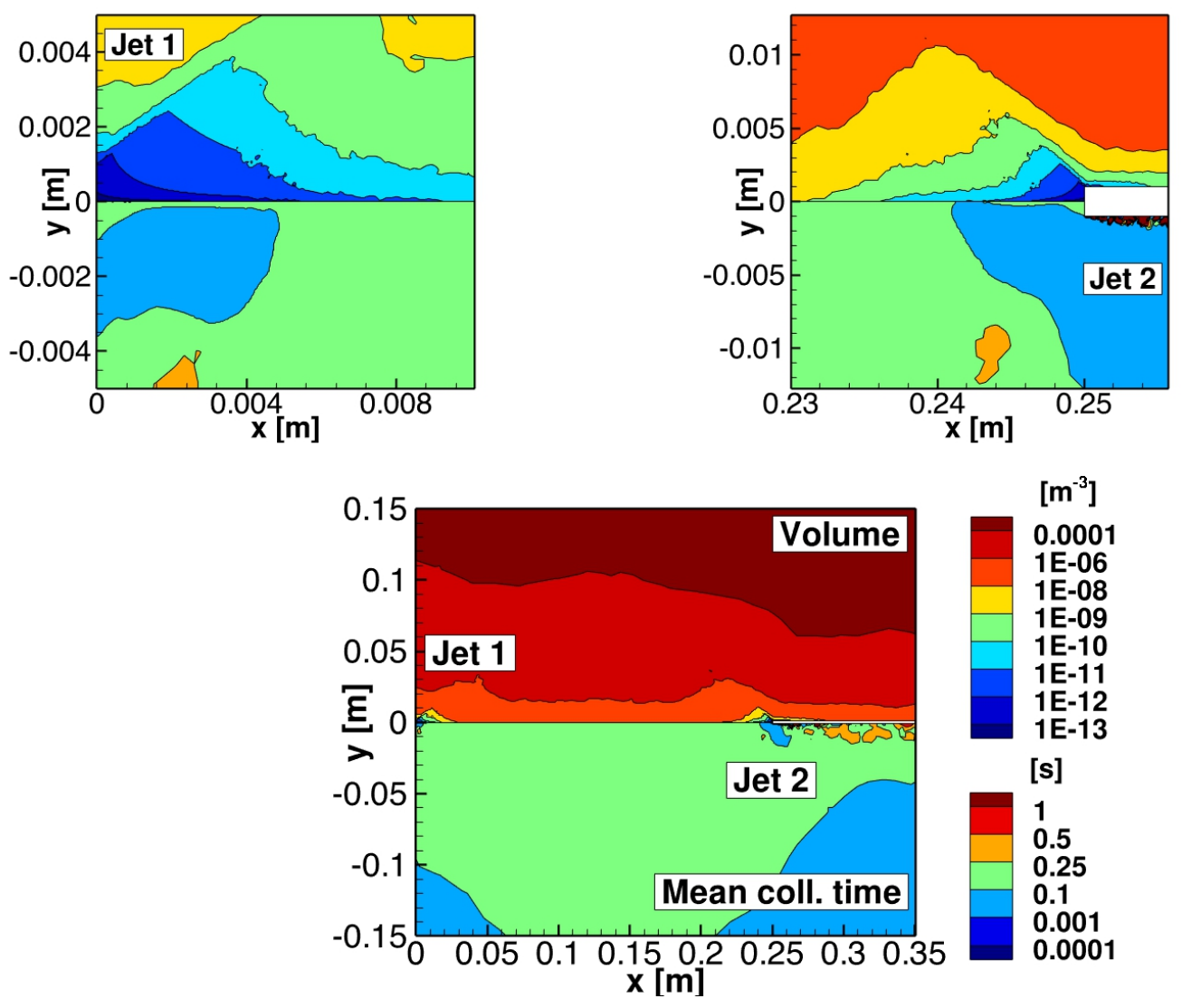

Figure 5: Cell volume $\mathrm{V}$ of the mesh employed (top) and mean collision time $\tau_{m}$.

by the usually small low Knudsen number zone thus accelerating the march to steady state of the simulation while increasing the convergence of sampled quantities in zones where the time step is increased. The value of the weight and the time step is often determined a priori which requires that the simulation be run at least twice to determine optimum time step and weight fields. The adaptive procedure presented in this work removes this need thus allowing simulations to be conducted with less human intervention. This is in particular useful for parametric studies where a large number of simulations are run on the same mesh with different boundary conditions or to allow non-expert DSMC users to run efficient simulations. In order to obtain a fair assessment of the efficiency gain of the adaptive method within the framework of this test case, the results of the adaptive method should be compared to those obtained with spatially varying time step and weights, as would almost certainly be used with most DSMC codes to simulate the present test case. The most natural time step and weight fields to use in the comparison are those that are themselves obtained by the adaptive method when applied to the test case. However, using these without relative weights (such as those produced by the adaptive method) results in a drop in the total number of particles in the simulation as relative weights naturally increase particle count in the cells for which they are less than 1. To compensate for this drop in the number of particles, $W_{p, 0}$ is reduced in the simulation so as to match the particle count produced by the adaptive method. The simulations conducted without relative weight, using the time step and weight field produced by the adaptive technique and with a suitably chosen $W_{p, 0}$, are designated as standard simulations in the following. The reference simulations corresponding to simulation A400 and A100, respectively, are designated by S400 and S100 in Table 2 where their numerical parameters are summarized. A comparison of the value of the sampled number density and velocity for 

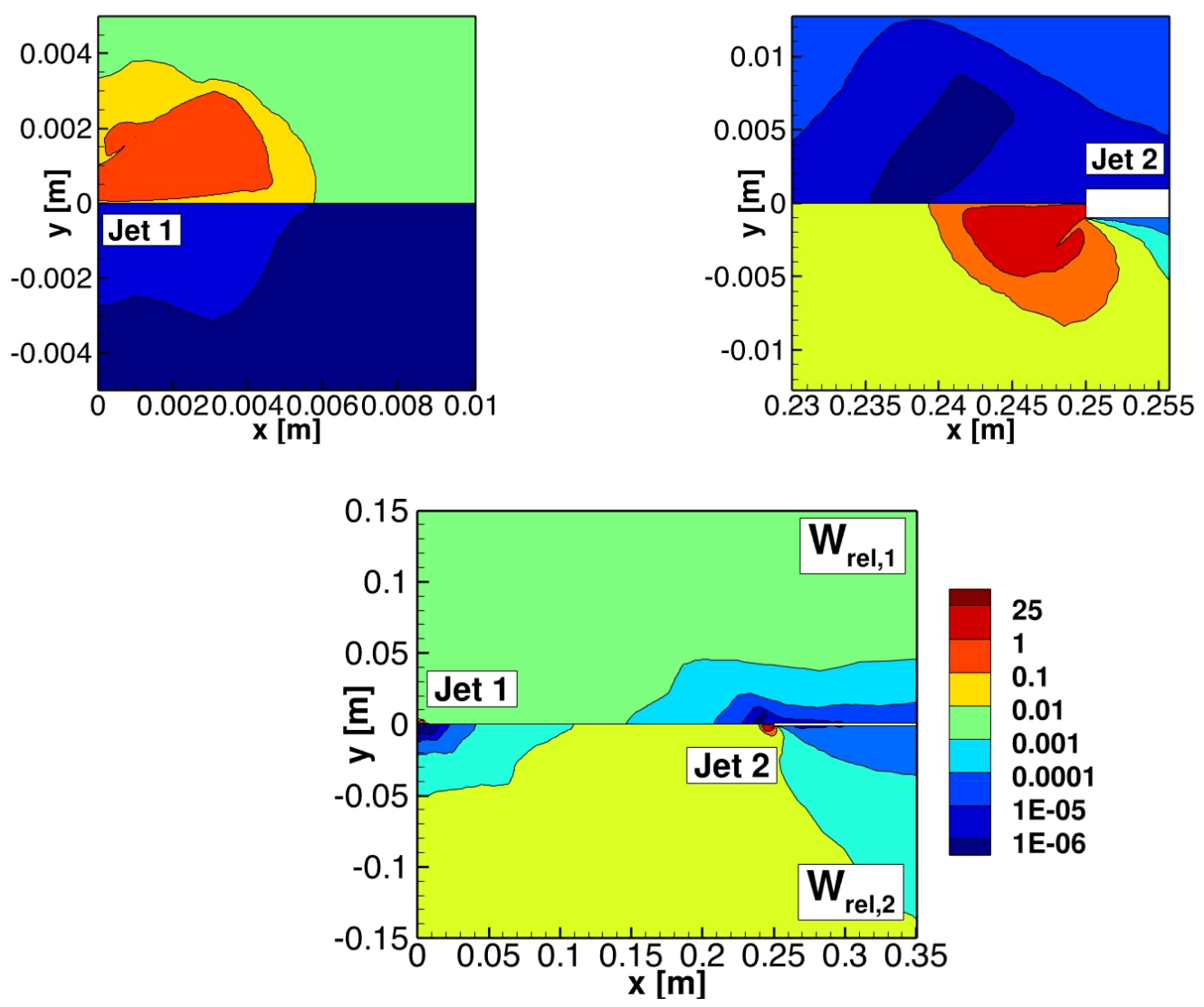

Figure 6: Relative weight for species $1, W_{\text {rel,1 }}$ (top) and 2, $W_{\text {rel,2 }}$ (bottom) for A400.

species 1 obtained with the standard (S100, S400) and adaptive methods (A100, A400) is presented in Figs. 10 and 11. The adaptive method is seen to produce a much smoother and more realistic sampled number density field than the standard method for both particle counts while an even greater improvement is visible for the sampled velocity. The adaptive method is thus seen to provide a large improvement over the standard method for the same particle count. This large qualitative improvement can be quantified by studying the numerical error of the various solutions plotted in Figs. 10 and 11.

We designate any cell-based quantity, such as number density or velocity of cell $i$, by $x_{i}$. The sampled value of $x_{i}$ is referred to by $\mu_{i}(x)$, while numerical error $\bar{\varepsilon}_{i}[\mu(x)]$ for a given simulation is defined by:

$$
\bar{\varepsilon}_{i}[\mu(x)] \triangleq \mu_{i}(x)-\mu_{i}^{E}(x),
$$

where $\mu_{i}^{E}(x)$ designates the exact solution, i.e. that obtainable in the limit of an infinite number of particles and an infinitesimally small time step. This definition of numerical error can in turn be used to define a normalized numerical error:

$$
\bar{\varepsilon}_{0, i}[\mu(x)]=\frac{\left|\bar{\varepsilon}_{i}[\mu(x)]\right|}{\left|\mu_{i}^{E}(x)\right|} .
$$

The exact solution for such a complex case is of course inaccessible in practice and must be approximated by that obtained with a much larger number of particles than the simulation for which the error is calculated. In the present study, the exact solution is thus assumed to be furnished by test case A4800 which is detailed in Table 3. Although one would ideally not want to use a solution obtained with the adaptive method to gauge its accuracy, we are forced to do so as obtaining a solution of comparable quality without relative 

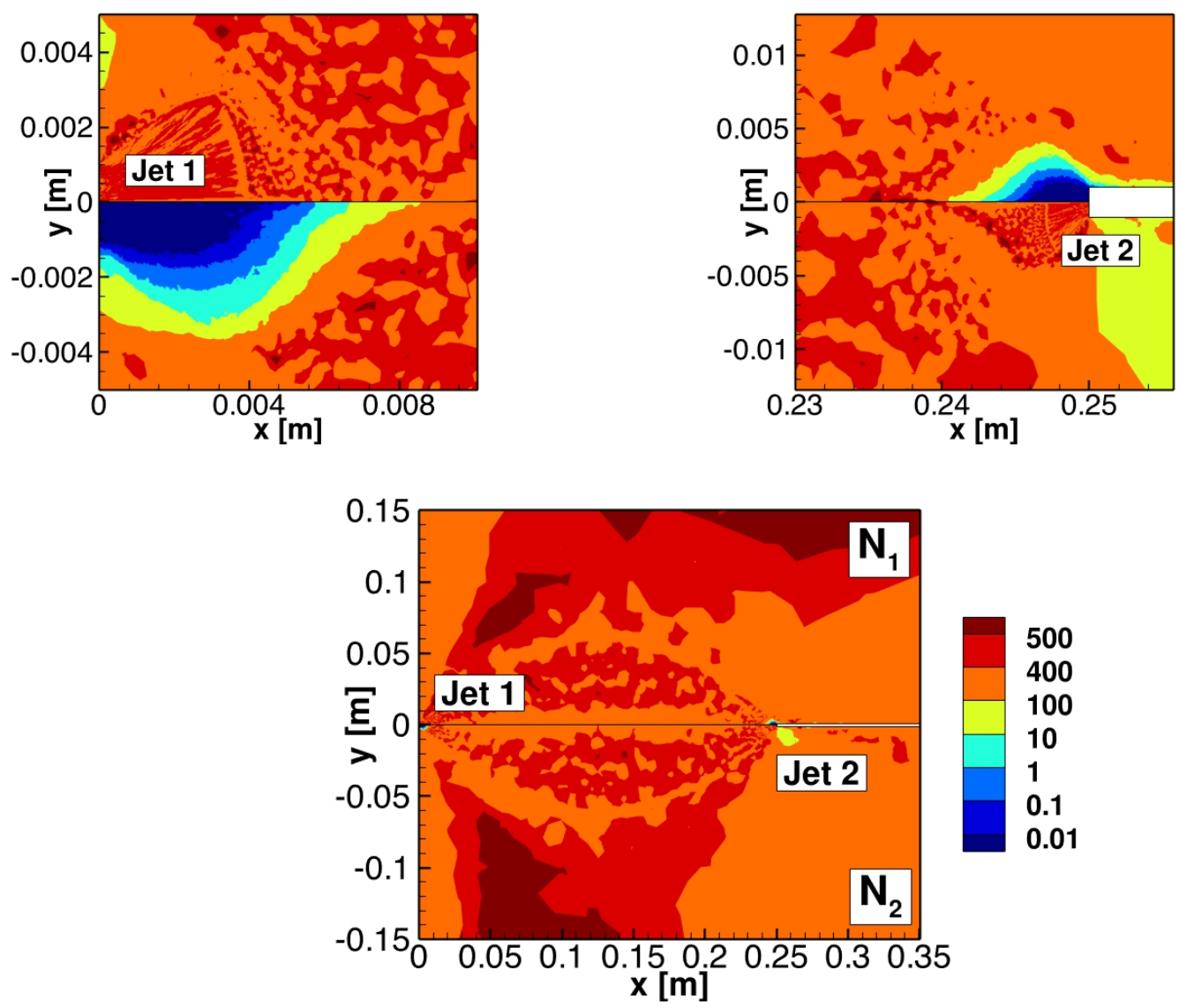

Figure 7: Average number of particles for species 1, $\bar{N}_{1}$ (top) and 2, $\bar{N}_{2}$ (bottom) for A400.

weights is prohibitively expensive. This, however, seems justified given the seemingly good results obtained with the adaptive method at lower $N_{p \text {,want }}$ values which are shown in Fig. 10. The normalized numerical error along the centerline for $\mu(n)$ and $\mu\left(V_{x}\right)$ obtained with both the adaptive and standard methods are plotted for species 1 in Fig. 12 and species 2 in Fig. 13. The log scale and the scaling of the $\mathrm{x}$ axis are chosen for each particular species so as to highlight the behavior of the error close to the jet opposite that from which it is emitted, where the error is highest. The adaptive method is observed to systematically produce a solution with a smaller numerical error than the standard method. An order of magnitude reduction in the value of the error for sampled number density and velocity is observed for both species for most of the centerline. The adaptive procedure for the two values of $N_{p \text {,want }}$ examined is, however, still not able to obtain accurate sampled quantities in the remaining $\Delta L \sim 0.005 \mathrm{~m}$. Proper resolution of the zone requires a further increase in $N_{p \text {,want }}$ such as that used to calculate the "exact" solution. The default value of the sampled number density and velocity in a cell is 0 , and both remain unchanged until at least one particle enters it. This explains the value of 1 for the normalized error that is observed at some points close to the opposing jet with the standard method which sometimes proves to be smaller than that obtained with the adaptive method. Similar improvements (not shown here) are obtained with the adaptive procedure for species 3 along the centerline as well as for sampled collision rates between species.

The large improvement observed for sampled quantities with the adaptive method for the same particle count can be explained by the improved spatial distribution of particles amongst the cells of the domain. 


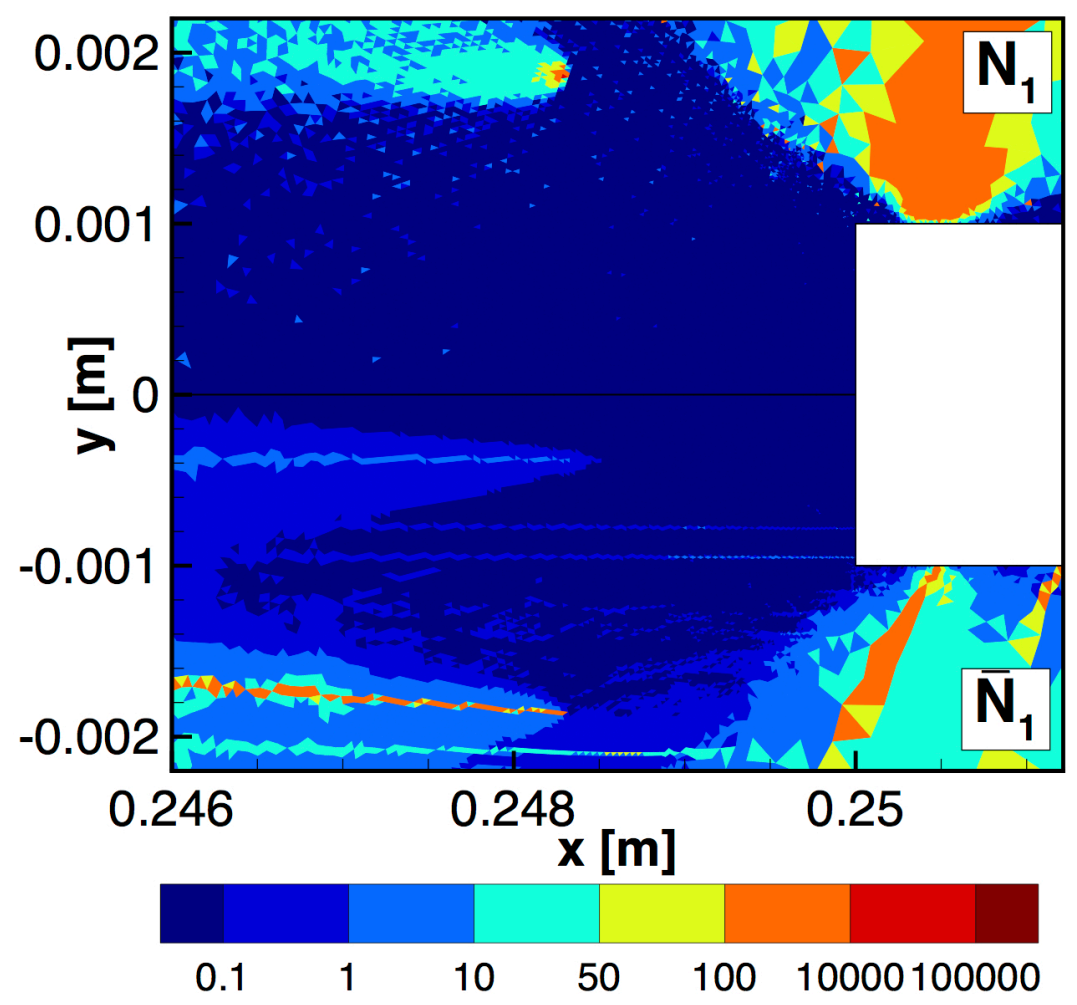

Figure 8: Closeup view of jet 2: Instantaneous number of particles per cell, $N_{1}$, (top) and average number of particles per cell, $\bar{N}_{1}$, (bottom) for species 1 at a given time step obtained with test case A100-1em10 demonstrating the occurrence of the avalanching phenomenon.

Figure 14 illustrates this point, showing that in the case S400, a few cells close to the inlet contain a large average number of particles of species 1 while that number rapidly decreases in the $x$ direction. In contrast to that, the average number of particles for test case A400 is much more uniform with no cell containing large, e.g. 1000, average numbers of particles, and only drops in close proximity to jet 2 . The situation can be summarized by the histogram presented in Fig. 15 which shows the distribution of all the cells of the domain according to their $\bar{N}_{1}$ value for both A400 and S400. In test case S400, the mean value of $\bar{N}_{1}$ is greater than that observed for S400, reflecting the fact that the simulation actually contains more particles of species 1 than A400 (18.6 vs $12.9 \mathrm{M}$ as indicated in Table 2). Whereas the distribution of $\bar{N}_{1}$ for A400, rapidly falls off for large $\bar{N}_{1}$ values, that for S400 displays a non-negligible tail (i.e. $\sim 14,000$ cells having $\bar{N}_{1} \in(800,1800]$ ). That tail corresponds to the cells located close to the inlet of jet 1 in Fig. 14, shown in dark red. The presence of a large number of particles in those cells amounts to a waste of computational effort as these particles do little to increase the accuracy of the simulation further downstream, in particular close to the second jet, since they quickly move away from the centerline. In the case of species 1 , the adaptive procedure thus both prevents cells close to jet 1 from containing an excessive number of particles while ensuring that the number of particles remains constant in the downstream direction which, in the present case, can be accomplished with fewer particles than with test case S400. The exact same phenomenon occurs for species 2 while the adaptive procedure, in the case of A400, because of the choice of $N_{p \text {,want, } 3}=400$, creates many more particles of species 3 (12.3 vs $0.19 \mathrm{M}$ as indicated in Table 2) than are present in the S400 simulation. This in turn suggests that the adaptive method will be of greatest benefit for DSMC simulations with a U-shaped distribution of the number of particles between cells such as that of S400 in Fig. 15. 


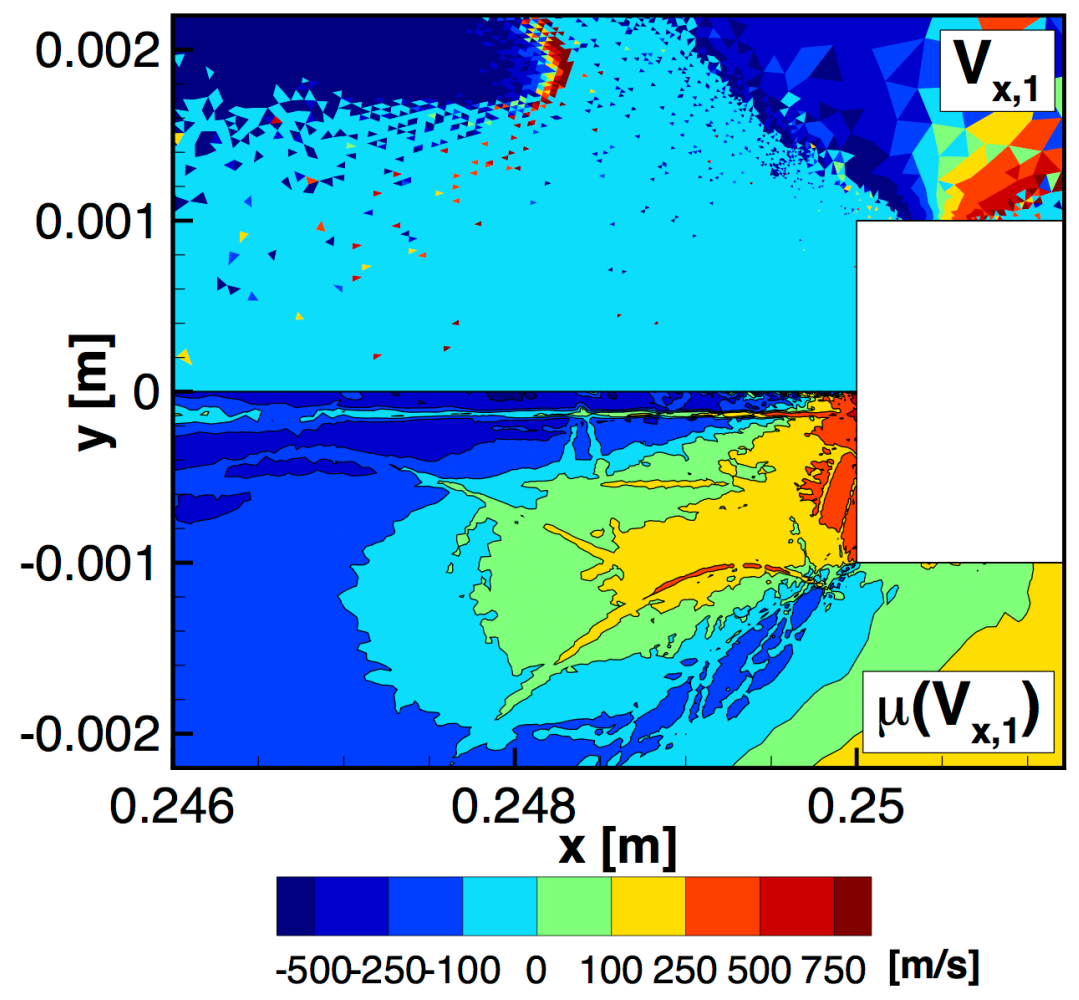

Figure 9: Closeup view of jet 2: Average velocity of particles of species 1 in each individual cell $\bar{V}_{x, 1}$ at a given time step, with $\bar{V}_{x, 1}=0$ in the absence of particles (top) and sampled $V_{x, 1}$ velocity (bottom) obtained with test case A100-1em10.

\section{Conclusions}

An adaptive method for the DSMC time step, cell weight and species relative weights was introduced in this article. The aim of the procedure presented is to automatically update the time step, weight and relative weights of all cells as the simulation progresses to steady state. The value of the time step is maximized while still requiring it to satisfy DSMC requirements for all cells. The value of the weights and relative weights are chosen so as to obtain a specified average number of particles for all species in all cells. The formulation of the adaptive method makes its implementation in existing DSMC codes straightforward while its computational overhead, compared to that of the simulation is negligible. It furthermore reduces the need for user input by determining the optimum time step and weight fields automatically thereby eliminating the need to run multiple simulations so as to determine them. The potential computational savings allowed by the method were illustrated by simulating the flow between two counterflowing jets at a Knudsen number of 0.015 with the new method on one hand and with a spatially varying time step and cell weight field on the other. For the same total number of particles, the use of the adaptive method allows a substantial reduction, i.e. an order of magnitude between the two jets, in the value of the error for the sampled number density and velocity. The increased computational efficiency is achieved by more uniformly distributing particles throughout the domain thereby preventing cells from containing an excessive or an insufficient number of particles. The adaptive method is thus expected to be of most benefit for multi-species flows with uneven distributions of the number of particles between cells and species. A deleterious consequence of the effect of particle cloning in the relative absence of collisions, the avalanching phenomenon, was examined and found to be mitigated by reducing the range of weights in the simulation while increasing the required average number of particles per cell. This work has furthermore highlighted the potential harmful effects introduced 


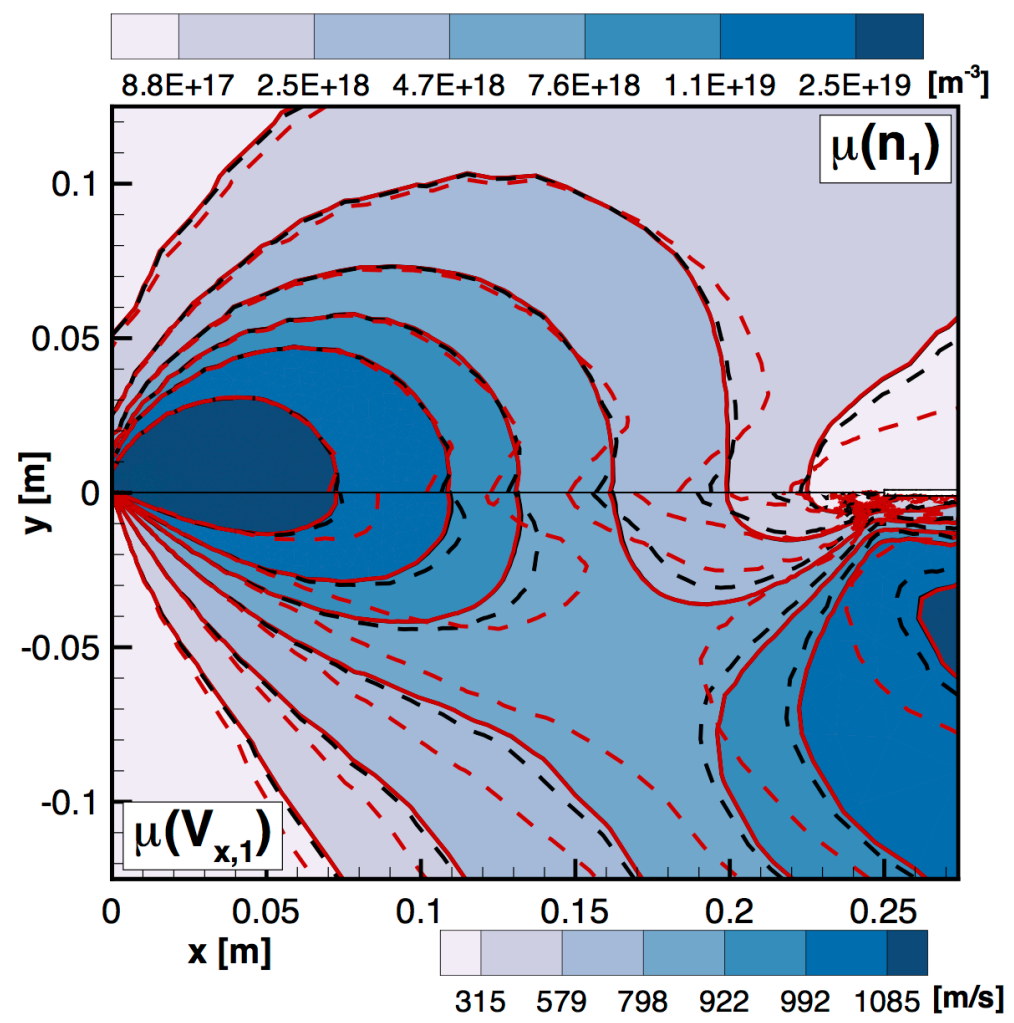

Figure 10: Sampled number density (top) and $V_{x, 1}$ velocity (bottom) obtained with the adaptive (solid) and standard method (dashed) with A or S400 (black) and A or S100 (red).

by the cloning of particles at weight interfaces. Alleviating those, by for instance partially delaying the introduction of clones at weight interfaces, could improve the accuracy of the present method and allow the use of even fewer particles. Finally, relative weights for particles could be extended beyond the species type to for instance refer to the internal energy. In particular, a slight modification of the adaptive procedure presented in the article could be used to more accurately and efficiently simulate the entire vibrational temperature range.

\section{Acknowledgment}

The authors gratefully acknowledge financial support from the Department of Energy through grant DE-SC0001939. CG wishes to thank Dr. Erin Farbar for useful comments.

\section{Appendix A. Avalanching phenomenon}

The avalanching phenomenon can best be understood by considering a simplified one-dimensional cold gas flowing with a bulk velocity of $V_{x}$ with a number density that decreases in the $x$ direction. As a Gedankenexperiment, we consider a one-dimensional perfectly cold gas flow in which the number density drops as $\sim x^{-1}$ so that its velocity distribution function is given by $f\left(V_{x}\right)=n_{0}\left(\frac{x_{0}}{x}\right) \delta\left(V_{x}-V_{0}\right)$, where $\delta$ is Dirac's delta function and $V_{0}>0$ while $n_{0}$ and $x_{0}$ designate a reference number density and length respectively. The gas flow is discretized with a one dimensional mesh from $x=0$ to $x=L$ with uniform spacing $\Delta x=\frac{L}{N_{c}}$ using $N_{c}$ cells each of volume $\mathrm{V}$ and constant time step $\Delta t_{0}$ is used (so that $\hat{W}_{p}=\tilde{W}_{p}$ ). 


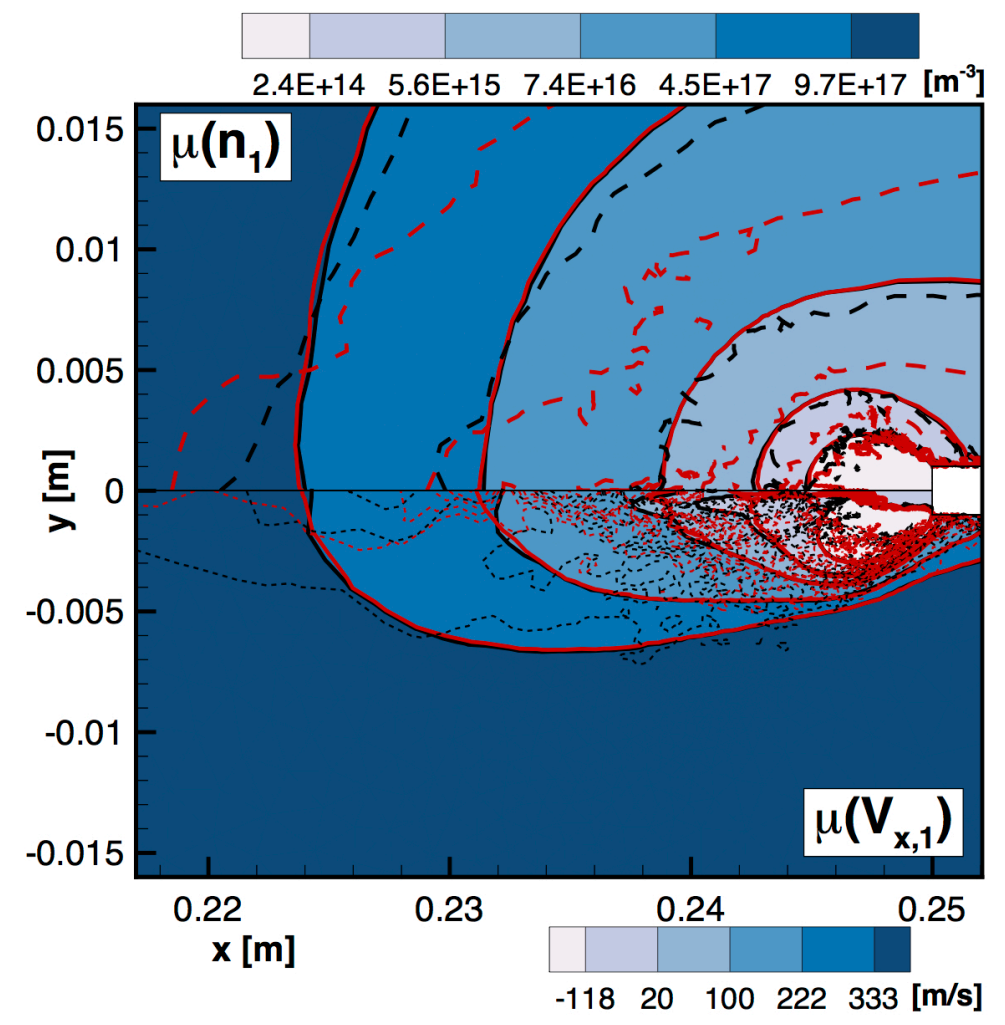

Figure 11: Closeup view of jet 2: Sampled number density (top) and $V_{x, 1}$ velocity (bottom) obtained with the adaptive (solid) and standard method (dashed) with A or S400 (black) and A or S100 (red). The A100 (solid red line) and A400 (solid black line) results are almost indistinguishable in most of the domain.

The weights of the cells are "naively" chosen so as to maintain a constant average number of particles $\bar{N}_{0}$ in all cells of the domain despite the drop in number density in the $x$ direction. The cell-averaged number density $\underline{\mathrm{n}}_{i}$ in cell $i$, characterized by $x_{i-1 / 2}<x<x_{i+1 / 2}$, is defined as $\underline{\mathrm{n}}_{i} \triangleq \frac{1}{\Delta_{x}} \int_{x_{i-1 / 2}}^{x_{i+1 / 2}} n_{0}\left(\frac{x_{0}}{x}\right) d x=$ $\frac{n_{0} x_{0}}{\Delta x} \log \left(\frac{x_{i+1 / 2}}{x_{i-1 / 2}}\right)$. Following Eq. (3), $\underline{\mathrm{n}}_{i}=\frac{W_{p, i} \bar{N}_{i}}{V}$, where $\bar{N}_{i}$ designates the average number of particles in cell $i$, the weights are thus given by $W_{p, i}=\frac{\mathrm{n}_{i} V}{\bar{N}_{0}}$. As was previously explained, due to the spatial variation of the cell weights, when a particle travels from cell $i$ to cell $i+1, \frac{\widetilde{W}_{p, i}}{\widetilde{W}_{p, i+1}}$ particles are created on the $i+1$ side of the interface between the two cells. Thus if a single particle happens to make its way from cell 1 to cell i, $\frac{\widetilde{W}_{p, 1}}{\widetilde{W}_{p, 2}} \times \frac{\widetilde{W}_{p, 2}}{\widetilde{W}_{p, 3}} \times \ldots \times \frac{\widetilde{W}_{p, i-1}}{\widetilde{W}_{p, i}}=\frac{\widetilde{W}_{p, 1}}{\widetilde{W}_{p, i}}$ identical particles are in turn obtained in cell $i$. Because cell $i$ has a lower density than cell 1 , such a large number of particles will only seldom be observed in cell $i$ which will contain no particles for most time steps. The frequency of occurrence of such a packet of particles in cell $i$, can be obtained by basic probability considerations. We designate the event "particle $\mathbf{p}$ is in cell $i+1$ in at least some time step $k$ " by $\omega_{\mathbf{p} \in i}$ and the event "particle $\mathbf{p}$ is in cell $i+1$ in at least some time step k" by $\omega_{\mathbf{p} \in i+1}$. Due to the simple nature of the flow considered, $\mathbf{p}$ has to pass through cell $i$ to reach cell $i+1$ so that $P\left(\omega_{\mathbf{p} \in i} \cap \omega_{\mathbf{p} \in i+1}\right)=P\left(\omega_{\mathbf{p} \in i+1}\right)$ and furthermore, by definition $P\left(\omega_{\mathbf{p} \in i+1} \mid \omega_{\mathbf{p} \in i}\right)=\frac{P\left(\omega_{\mathbf{p} \in i} \cap \omega_{\mathbf{p} \in i+1}\right)}{P\left(\omega_{\mathbf{p} \in i}\right)}$ so that $P\left(\omega_{\mathbf{p} \in i+1} \mid \omega_{\mathbf{p} \in i}\right)=\frac{P\left(\omega_{\mathbf{p} \in i+1}\right)}{P\left(\omega_{\mathbf{p} \in i}\right)}$. It can readily be seen that $P\left(\omega_{\mathbf{p} \in i+1} \mid \omega_{\mathbf{p} \in i}\right)=\frac{\underline{\underline{n}}_{i+1}}{\underline{\underline{n}}_{i}}$ so that $P\left(\omega_{\mathbf{p} \in i}\right)=$ $P\left(\omega_{\mathbf{p} \in i} \mid \omega_{\mathbf{p} \in i-1}\right) \times P\left(\omega_{\mathbf{p} \in i-1} \mid \omega_{\mathbf{p} \in i-2}\right) \times \ldots \times P\left(\omega_{\mathbf{p} \in 2} \mid \omega_{\mathbf{p} \in 1}\right)=\frac{\underline{\mathrm{n}}_{i}}{\underline{\mathrm{n}}_{i-1}} \times \frac{\underline{\underline{\mathrm{n}}}_{i-1}}{\underline{\underline{\mathrm{n}}}_{i-2}} \times \ldots \times \frac{\underline{\underline{\mathrm{n}}}_{2}}{\underline{\underline{n}}_{1}}=\frac{\underline{\underline{\mathrm{n}}}_{i}}{\underline{\underline{\mathrm{n}}}_{1}}$. Hence a packet of $\frac{\widetilde{W}_{p, 1}}{\widetilde{W}_{p, i}}$ particles will occur with a probability of $\frac{\underline{\mathrm{n}}_{i}}{\underline{\underline{1}}_{1}}$, i.e. once every $\frac{\underline{\mathrm{n}}_{1}}{\underline{\underline{\underline{n}}}_{i}}$ steps in cell $i$. Thus, cell $i$ will indeed 


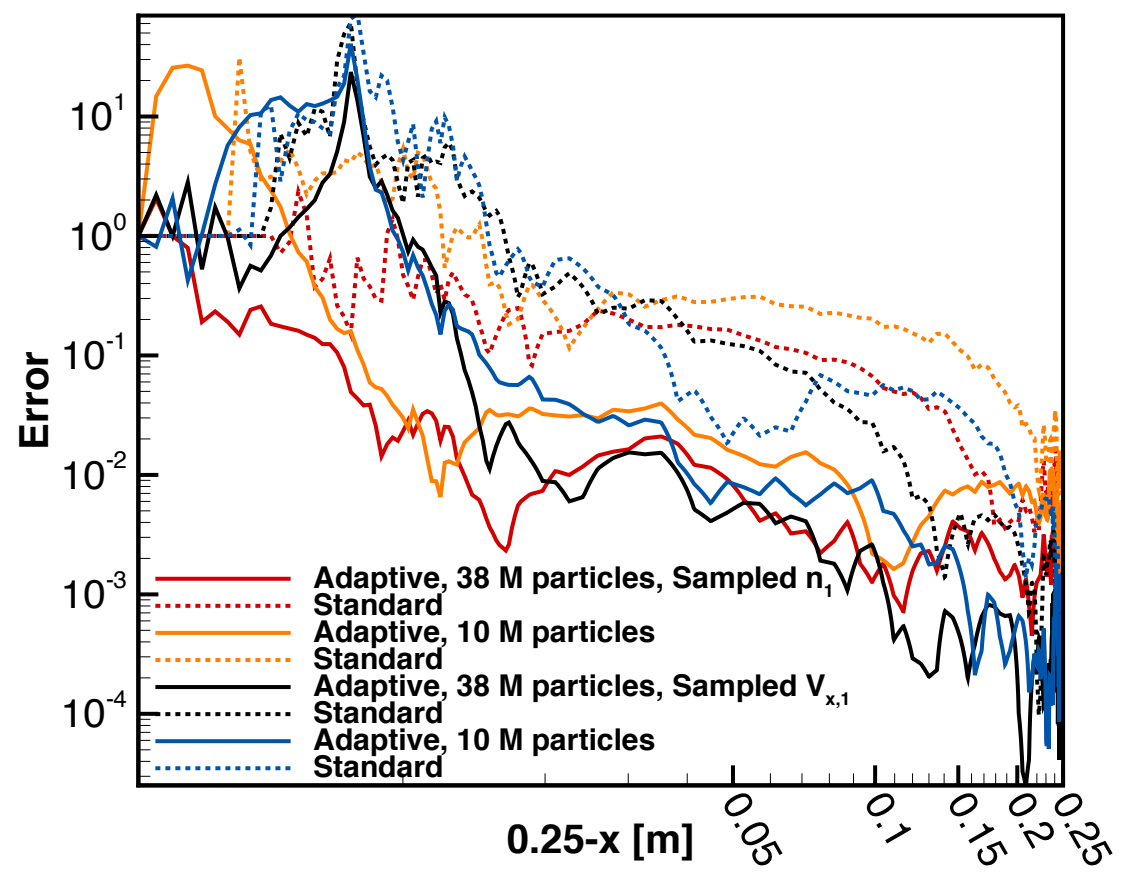

Figure 12: Numerical error for sampled $n_{1}$ and $V_{x, 1}$ on the centerline computed with A4800 as an exact solution.

contain an average of $N_{0} \times \frac{\underline{n}_{i}}{\underline{\underline{n}}_{1}} \times \frac{\widetilde{W}_{p, 1}}{\widetilde{W}_{p, i}}=N_{0}$ over the course of the simulation as the process described above will be repeated $N_{0}$ times for all the particles initially in cell 1 . This though experiment illustrates how decreasing weights to offset a decrease in the number density in one direction combined with the relative absence of collisions can create a large number of identical particles that travel together. This numerical phenomenon is referred to as an avalanching phenomenon in this article.

\section{References}

[1] I. Boyd, Direct simulation Monte Carlo for atmospheric entry. 2. Code development and application results. NATO Conf. Proc. RTO-EN-AVT-162, 2009.

[2] G. Bird, Molecular Gas Dynamics and the Direct Simulation of Gas Flows, Oxford University Press, 1994.

[3] Y. Choi, I. Boyd, M. Keidar, Effect of a magnetic field in simulating the plume field of an anode layer Hall thruster, Journal of Applied Physics 105 (2009).

[4] I. Boyd, Modeling of associative ionization reactions in hypersonic rarefied flows, Physics of Fluids 19 (2007).

[5] D. Economou, T. Bartel, R. Wise, D. Lymberopoulos, Two-dimensional direct simulation monte carlo (DSMC) of reactive neutral and ion flow in a high density plasma reactor, IEEE Transactions on Plasma Science 23 (1995) 581-590.

[6] I. Boyd, Conservative species weighting scheme for the direct simulation Monte Carlo method, Journal of Thermophysics and Heat Transfer 10 (1996).

[7] K. Kannenberg, I. Boyd, Strategies for Efficient Particle Resolution in the Direct Simulation Monte Carlo Method, Journal of Computational Physics 157 (2000) 727-745.

[8] R. Kumar, Z. Li, D. Levin, Modeling of $\mathrm{CO} 2$ condensation in the high pressure flows using the statistical BGK approach, AIAA Paper 2010-818, 2010.

[9] D. Olynick, H. Hassan, J. Moss, Grid generation and adaptation for the direct simulation Monte Carlo method, Journal of Thermophysics and Heat Transfer 3 (1989) 368-373.

[10] M. Laux, Local time stepping with automatic adaptation for the DSMC method, AIAA Paper 98-2670, 1998. 


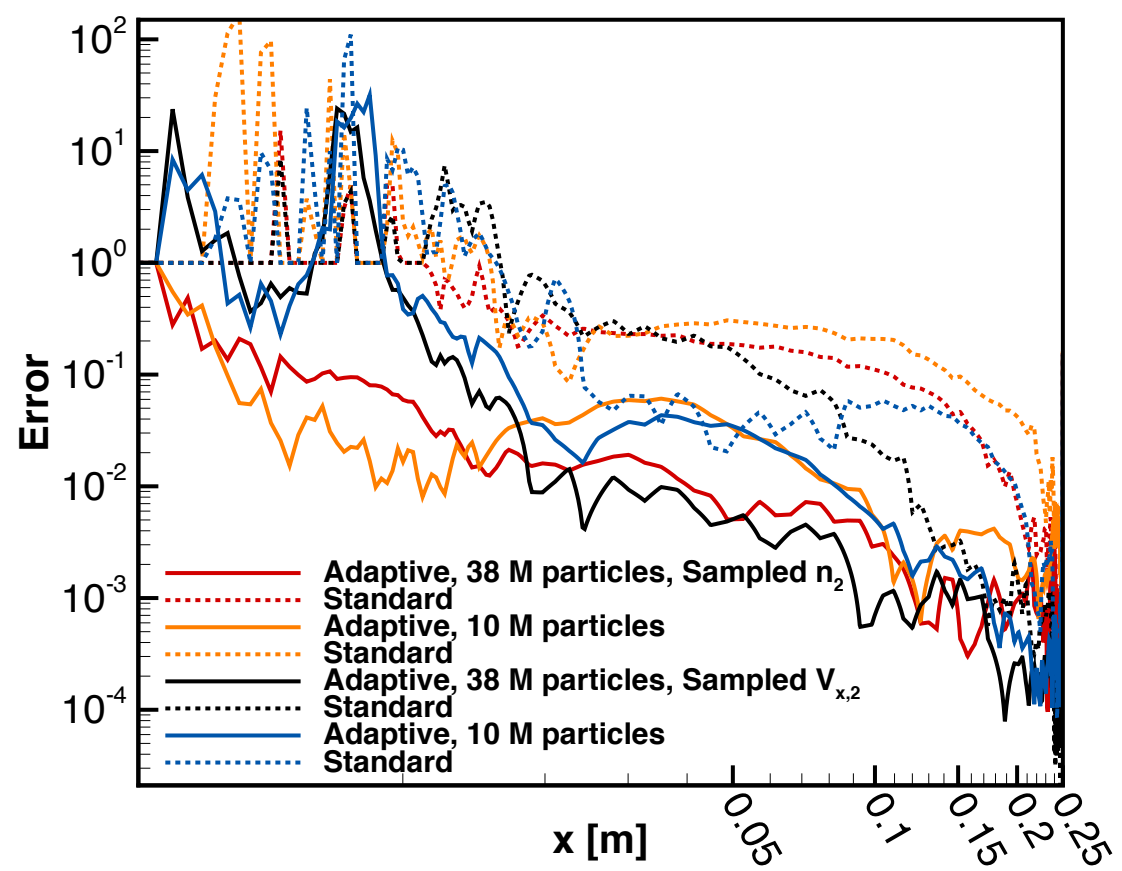

Figure 13: Numerical error for sampled $n_{2}$ and $V_{x, 2}$ on the centerline computed with A4800 as an exact solution.

[11] G. Bird, M. Gallis, J. Torczynski, D. Rader, Accuracy and efficiency of the sophisticated direct simulation Monte Carlo algorithm for simulating noncontinuum gas flows, Physics of Fluids 21 (2009) 017103

[12] M. Bartel, J. Johannes, T. Furlani, Trace chemistry modelling with DSMC in chemically reacting plasmas, AIAA Paper 98-2753, 1998.

[13] A. Alexeenko, S. Gimelshein, D. Levin, A. Ketsdever, M. Ivanov, Measurements and simulation of orifice flow for micropropulsion testing, Journal of Propulsion and Power 19 (2003) 588-594.

[14] Q. Sun, I. Boyd, Evaluation of macroscopic properties in the direct simulation Monte Carlo method, Journal of Thermophysics and Heat Transfer 19 (2005) 329-335.

[15] S. Piersall, J. Anderson, Direct Monte Carlo simulation of bimolecular reactions of chemical reaction systems: Simple bimolecular reactions, Journal of Chemical Physics 95 (1991) 971-978.

[16] S. Rjasanow, W. Wagner, Stochastic Numerics for the Boltzmann Equation, Springer, 2005.

17] G. Bird, Molecular Gas Dynamics, Clarendon Press, 1976.

[18] J. Wu, W. Hsiao, Y. Lian, K. Tseng, Assessment of conservative weighting scheme in simulating chemical vapour deposition with trace species, International Journal for Numerical Methods in Fluids 114 (2003) 93-114.

[19] G. Bird, The DSMC Method, Create Space Independent Publishing Platform, 2013.

[20] C. Galitzine, On the Accuracy and Efficiency of the Direct Simulation Monte Carlo Method, Ph.D. thesis, University of Michigan, 2014.

[21] C. Galitzine, I. Boyd, Simulation of the interaction between two counterflowing rarefied jets, in: Proceedings of the 26th International Symposium on Rarefied Gas Dynamics, AIP, 2011, pp. 545-550.

[22] S. Dietrich, I. Boyd, Scalar and parallel optimized implementation of the direct simulation Monte Carlo method, Journal of Computational Physics 126 (1996) 328-342. 


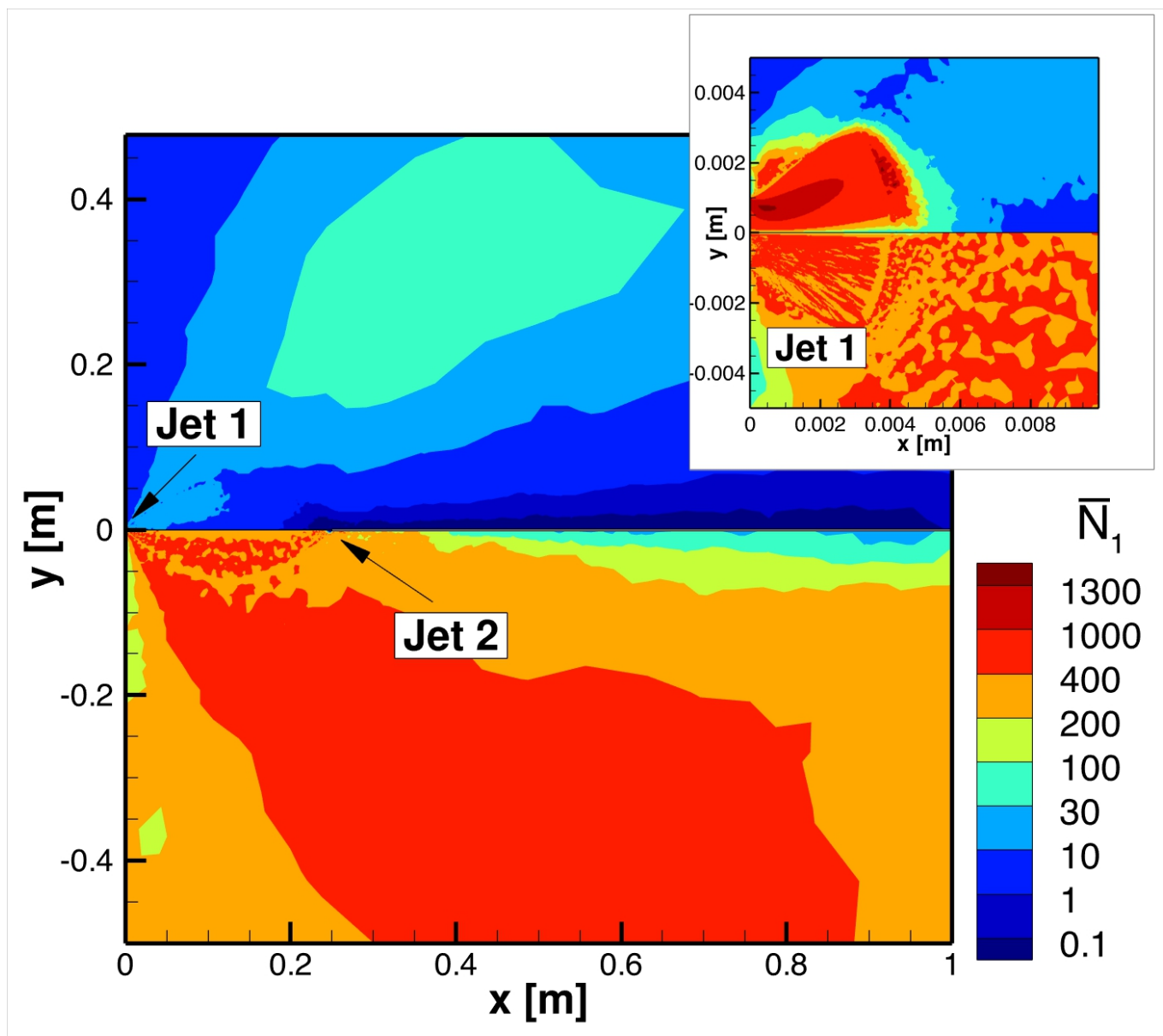

Figure 14: Comparison of the average number of particles for species $1, \bar{N}_{1}$, obtained for test case S400 (top) and for test case A400 (bottom). Closeup view shows situation close to jet 1. 


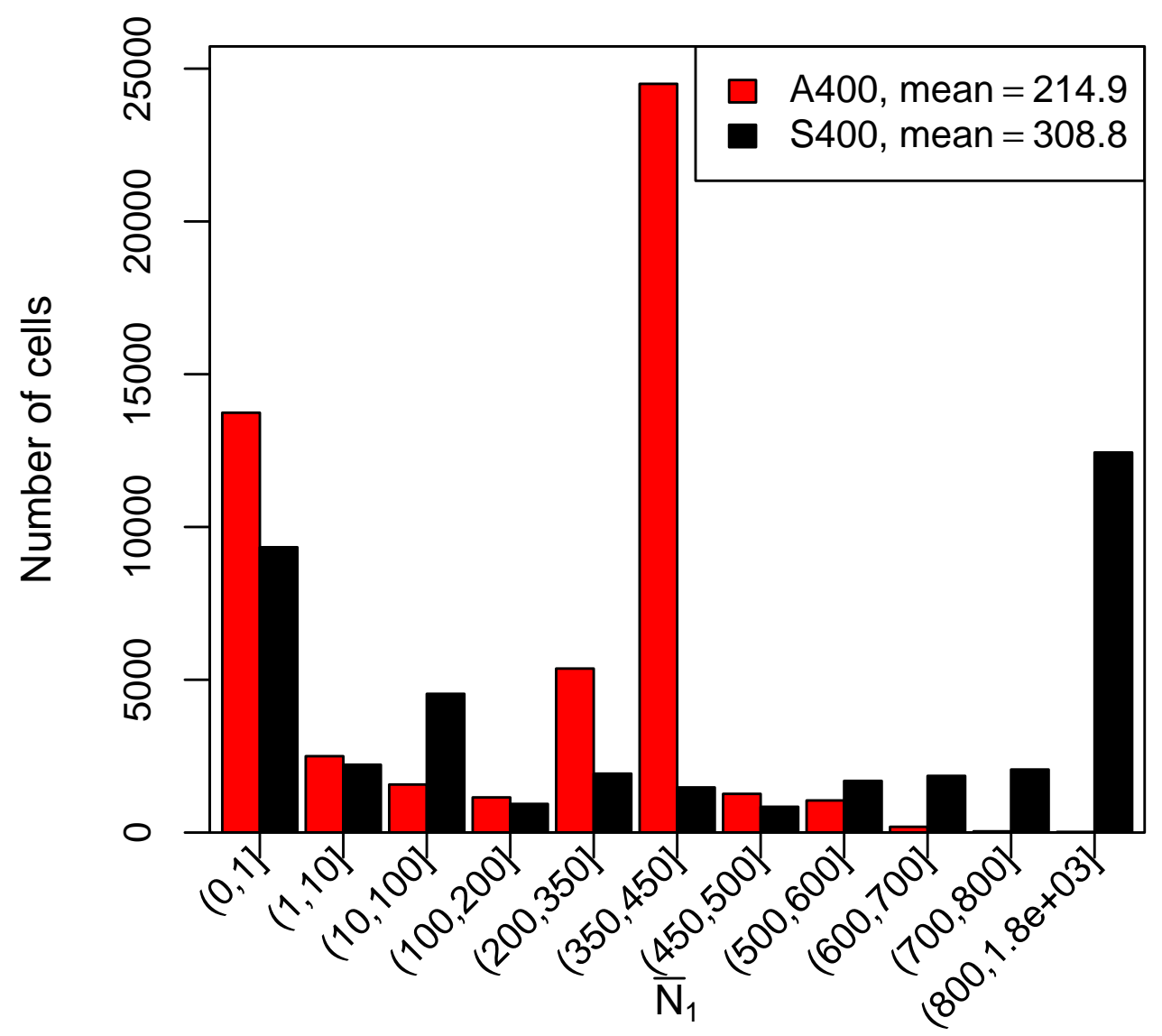

Figure 15: Comparison of the distribution of particles of species 1 amongst the cell of the domain for test cases A400 and S400. 